\title{
Activation of neutral sphingomyelinase 2 through hyperglycemia contributes to endothelial apoptosis via vesicle-bound intercellular transfer of ceramides
}

\author{
Andreas Zietzer ${ }^{1}$ (D) Alina Lisann Jahnel ${ }^{1} \cdot$ Marko Bulic $^{1} \cdot$ Katharina Gutbrod $^{2} \cdot$ Philip Düsing $^{1}$. \\ Mohammed Rabiul Hosen ${ }^{1} \cdot$ Peter Dörmann ${ }^{2} \cdot$ Nikos Werner $^{1,3} \cdot$ Georg Nickenig ${ }^{1} \cdot$ Felix Jansen ${ }^{1}$
}

Received: 23 April 2021 / Revised: 12 November 2021 / Accepted: 18 November 2021 / Published online: 24 December 2021

(c) The Author(s) 2021

\begin{abstract}
Background Pro-apoptotic and pro-inflammatory ceramides are crucially involved in atherosclerotic plaque development. Local cellular ceramide accumulation mediates endothelial apoptosis, especially in type 2 diabetes mellitus, which is a major cardiovascular risk factor. In recent years, large extracellular vesicles (lEVs) have been identified as an important means of intercellular communication and as regulators of cardiovascular health and disease. A potential role for lEVs as vehicles for ceramide transfer and inductors of diabetes-associated endothelial apoptosis has never been investigated.

Methods and Results A mass-spectrometric analysis of human coronary artery endothelial cells (HCAECs) and their lEVs revealed C16 ceramide (d18:1-16:0) to be the most abundant ceramide in IEVs and to be significantly increased in lEVs after hyperglycemic injury to HCAECs. The increased packaging of ceramide into lEVs after hyperglycemic injury was shown to be dependent on neutral sphingomyelinase 2 (nSMase2), which was upregulated in glucose-treated HCAECs. 1EVs from hyperglycemic HCAECs induced apoptosis in the recipient HCAECs compared to native lEVs from untreated HCAECs. Similarly, IEVs from hyperglycemic mice after streptozotocin injection induced higher rates of apoptosis in murine endothelial cells compared to IEVs from normoglycemic mice. To generate IEVs with high levels of C16 ceramide, ceramide was applied exogenously and shown to be effectively packaged into the IEVs, which then induced apoptosis in IEV-recipient HCAECs via activation of caspase 3. Intercellular transfer of ceramide through $\mathrm{IEVs}$ was confirmed by use of a fluorescently labeled ceramide analogue. Treatment of HCAECs with a pharmacological inhibitor of nSMases (GW4869) or siRNA-mediated downregulation of nSMase2 abrogated the glucose-mediated effect on apoptosis in lEV-recipient cells. In contrast, for small EVs (sEVs), hyperglycemic injury or GW4869 treatment had no effect on apoptosis induction in sEV-recipient cells.

Conclusion IEVs mediate the induction of apoptosis in endothelial cells in response to hyperglycemic injury through intercellular transfer of ceramides.
\end{abstract}

Andreas Zietzer

andreas.zietzer@ukbonn.de

Felix Jansen

felix.jansen@ukbonn.de

1 Department of Internal Medicine II, University Hospital Bonn, University of Bonn, Venusberg-Campus 1,

53127 Bonn, Germany

2 Institute of Molecular Physiology and Biotechnology of Plants, University of Bonn, Karlrobert-Kreiten-Str. 13, 53115 Bonn, Germany

3 Krankenhaus der Barmherzigen Brüder Trier, Nordallee 1, 54292 Trier, Germany 


\section{Graphical abstract}

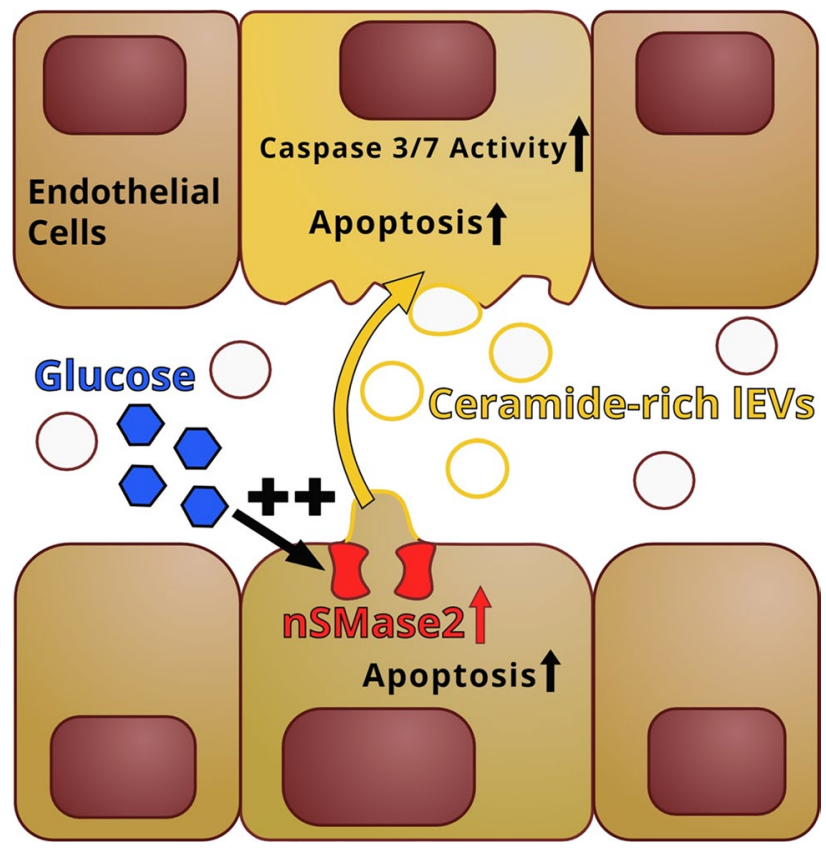

Keywords Extracellular vesicles $\cdot$ Diabetes $\cdot$ Endothelial cell $\cdot$ Apoptosis $\cdot$ Ceramide $\cdot$ SMPD3

\section{Background}

In recent years, sphingolipids have been shown to be important mediators of cardiovascular health and disease [1]. Elevated levels of ceramides in human plasma have been linked to an increased risk of cardiovascular events [2]. In particular, high levels of ceramides with a long carboxyl chain (e.g., C16 ceramide) have been shown to be associated with the development of cardiovascular disease and to be of prognostic value for the prediction of cardiovascular events [3]. On the cellular level, ceramides are produced in response to toxic stimuli and serve as potent pro-apoptotic and pro-inflammatory signaling molecules [4-6]. Generally, cellular ceramide production is governed by sphingomyelinases and ceramidases [7, 8]. Rapid generation of ceramide, in response to cellular injury from extracellular stressors, is mainly regulated by sphingomyelinases. Of these sphingomyelinases, isoform 3 (sphingomyelin phosphodiesterase $3=S M P D 3$, which codes for the protein neutral sphingomyelinase $2=n$ nMase2) is the most responsive isoform with regard to important inductors of atherosclerotic plaque development, such as oxidative stress, inflammation, and apoptosis $[9,10]$. Therefore, nSMase 2 activity has been shown to be involved in atherosclerotic plaque development in vivo [11]. Moreover, ceramides have been shown to be involved in the development of type 2 diabetes mellitus [12], which is one of the most important risk factors for cardiovascular disease [13, 14]. Diabetes-associated hyperglycemia also triggers atherosclerotic plaque development through the impairment of endothelial function and regeneration $[15,16]$. This again involves the induction of endothelial apoptosis through oxidative stress and the accumulation of ceramides [17-20].

Over the past decade, extracellular vesicles (EVs) have been identified as crucial regulators of atherosclerotic plaque development [21-24]. Virtually every kind of cell releases EVs, which can be taken up by both adjacent and distant cells $[25,26]$. Therefore, EVs have the potential to transport various molecules, such as nucleic acids and proteins, but also lipids [21]. The relatively small encapsulated volume, in relation to the large surface of the outer membrane, makes EVs interesting as lipid carriers, and the strong curvature of the EV membrane requires lipids with a specific head-groupto-tail size ratio [27]. Ceramides typically induce membrane curvature due to their conical shape, which is why the release of small EVs (exosomes) from the multivesicular endosome is dependent on the supply of ceramide provided by nSMases [28, 29]. For large EVs (lEVs, formerly called microvesicles), which are released via direct budding from the cytoplasmic membrane, the importance of the ceramide supply and sphingomyelinase activity remains controversial [30, 31]. In the context of cardiovascular health, however, IEVs are by no means less important than small EVs, as ours and other groups have shown [32-34]. 
In a recent study, ceramide levels from plasma-derived EVs have been identified as biomarkers of acute myocardial infarction [35]. Mechanisms of loading ceramides into lEVs and the biological function of IEVs as intercellular ceramide transporters has not yet been investigated in the context of cardiovascular disease. Likewise, the roles of cardiovascular risk factors such as type 2 diabetes mellitus on vesicular ceramide release and on the induction of ceramide-mediated apoptosis remain unclear. In this study, we show for the first time that lEV-bound intercellular ceramide transfer is a mediator of endothelial apoptosis under hyperglycemic conditions.

\section{Methods}

\section{Cell culture}

As our experimental model, we used male human coronary artery endothelial cells (HCAECs) from Promocell (Cat\# C-12221), which were cultured under standard conditions $\left(37{ }^{\circ} \mathrm{C}, 5 \% \mathrm{CO}_{2}, 100 \%\right.$ relative humidity) using Endothelial Cell Growth Medium MV (Promocell Cat\# C-22020). Experiments were performed with cells from passages 8-9. For IEV isolation, the HCAECs were cultured in serum-free medium for $24 \mathrm{~h}$, similar to previously published methods from ours and other groups [36-38]. Hyperglycemic injury was induced by the addition of a $20 \%$ glucose solution (glucose 20\%, B. Braun, Cat\# B05BA03) to achieve a supplement of $5 \mathrm{mM}$ and $30 \mathrm{mM}$ glucose to the regular medium, as previously reported by our and other groups [37, 39]. This model is clinically relevant, as blood glucose concentrations higher than $30 \mathrm{mM}$ can be observed in patients suffering from hyperglycemic crises, which is not a rare but severe complication of diabetes mellitus [40]. Additionally, hyperosmolar injury was simulated by the addition of a $15 \%$ mannitol solution (M15, Burg Pharma GmbH Cat\# 1021) to the regular medium, to achieve a supplement of $30 \mathrm{mM}$ mannitol. To generate lEVs with increased ceramide levels, we used exogenous C16 ceramide (d18:1/16:0, N-palmitoylD-erythro-sphingosine, Avanti Polar Lipids Cat\# 860516P, short $\mathrm{C16}$ ), because we found it to be the most abundant ceramide in HCAECs and HCAEC-derived lEVs. C16 ceramide was dissolved in dimethyl sulfoxide (DMSO, AppliChem $\mathrm{GmbH}$, Cat\# A3672,0100) at a concentration of $5 \mathrm{mM}$. The final concentrations used for the cell-culture experiments are indicated in the respective figures. GW4869, an inhibitor of nSMases, was dissolved in DMSO at a concentration of $5 \mathrm{mM}$ and used at concentrations of $5-10 \mu \mathrm{M}$, as previously reported [30, 41]. For all experiments with C16 or GW4869, equal amounts of DMSO were added to the control group. For experiments with murine endothelial cells, we used male C57BL/6 mouse primary carotid artery endothelial cells (MCAEC, Cell Biologics Cat\# C57-6008), which were cultured in complete mouse endothelial cell medium (Cell Biologics M1168).

\section{IEV isolation}

The isolation of $1 E V s$ was performed as previously reported by our group [32, 37, 42], using a three-step centrifugation protocol in an Eppendorf Centrifuge 5430 with an FA-4516-17 rotor. In brief, the culture supernatant was centrifuged at $1500 \times \mathrm{g}$ for $15 \mathrm{~min}$ to remove cellular debris. The lEVcontaining supernatant was then centrifuged at $20,000 \times g$ for $40 \mathrm{~min}$ at $4{ }^{\circ} \mathrm{C}$ to pellet the lEVs. The lEVs were washed by resuspending them in PBS and pelleted again at 20,000 $\times g$ for $40 \mathrm{~min}$ (Fig. 1A). For EV stimulation experiments, equal numbers of EV-releasing cells were used across the different conditions in the same experiment. We used IEVs from approximately $2.0 \times 10^{6}$ HCAECs after $24 \mathrm{~h}$ of serum-free culture and resuspended them in $2 \mathrm{ml}$ of culture medium. For untreated HCAECs, this procedure led to a concentration of approximately $5.0 \times 10^{8}$ endothelial cell-derived EVs per $\mathrm{ml}$. This corresponds to the $5-15 \%$ of endothelial-cellderived lEVs reported in the literature [43], with an estimation of $2.0 \times 10^{9}-1.0 \times 10^{10}$ total lEVs $(>100 \mathrm{~nm})$ in the peripheral blood [44-46].

\section{sEV isolation}

sEVs were isolated from the supernatant after IEVs had been removed, as described above, through a main centrifugation step at $100,000 \times g, 4{ }^{\circ} \mathrm{C}$ for $90 \mathrm{~min}$ in a Beckman Coulter Optima LE-80 K ultracentrifuge with a SW41Ti rotor, as previously reported $[47,48]$. Extensive characterization studies of endothelial cell-derived sEVs, isolated by this method, have been published previously by our group [48]. For stimulation experiments with sEVs, the same approach was performed as for $1 E V s$ but using sEVs from approximately $2.0 \times 10^{6}$ HCAECs in $2 \mathrm{ml}$ of culture medium, to allow for a comparison between IEVs and sEVs.

\section{Flow-cytometric analysis of apoptosis}

Early apoptosis in HCAECs was quantified by use of the FITC Annexin V Apoptosis Detection Kit with 7-AAD (Biolegend, Cat\# 640922). For this assay, we used 12-well plates at $90 \%$ confluence. The assay was carried out as recommended by the manufacturer. In brief, the cells were washed twice with PBS. Then the cells were carefully detached by use of the Detach kit (Promocell C-41210), centrifuged, and suspended in $100 \mu \mathrm{l}$ of Annexin V binding buffer. Subsequently, the FITC-Annexin V and the 7-AAD staining solutions were added ( $5 \mu \mathrm{l}$ of each), and the sample was incubated for $15 \mathrm{~min}$ at RT. Before analysis in a 
(A)

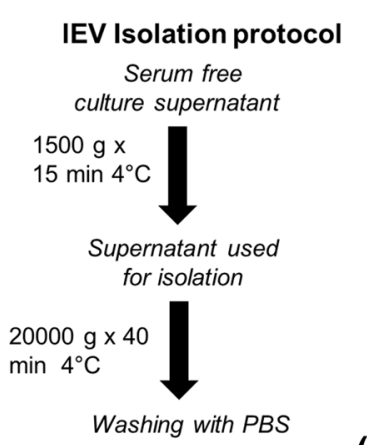

$20000 \mathrm{~g} \times 40$ $\min 4^{\circ} \mathrm{C}$

Immediate use of EV pellet for experiments
(B) Flow cytometric analysis

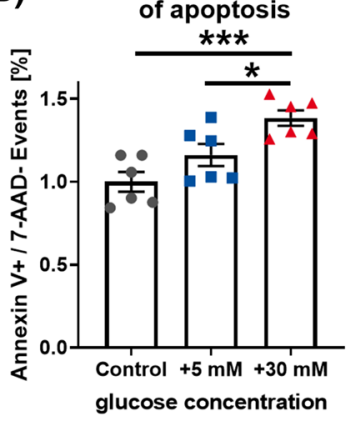

(C)

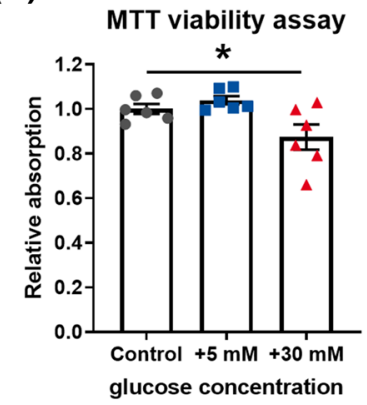

Representative dot blots

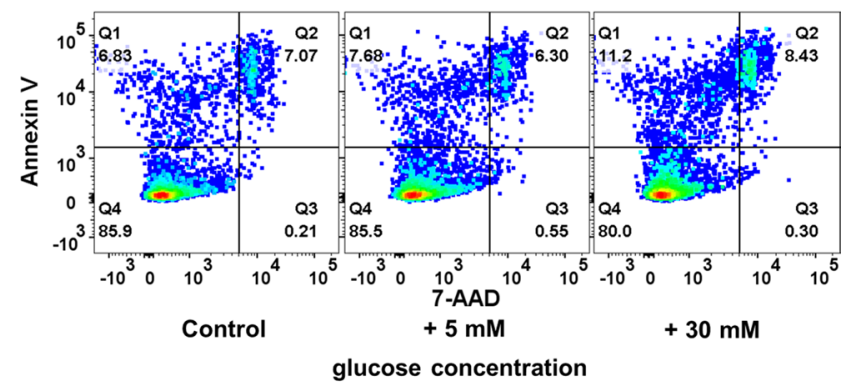

(D)

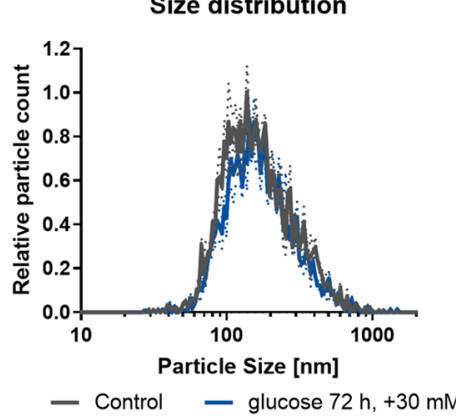

(E)

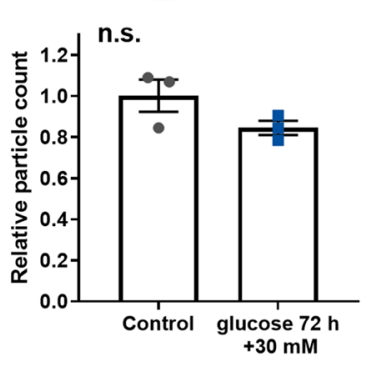

(F)

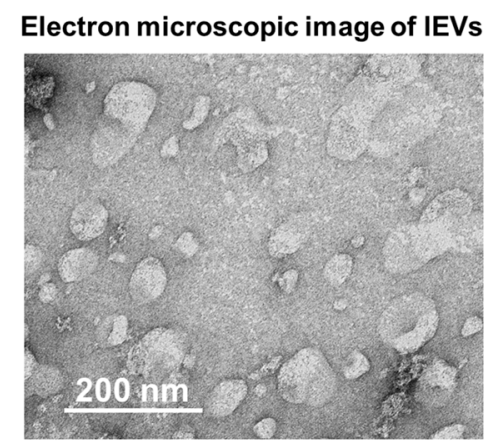

(G)

IEV characterization by Immunoblotting

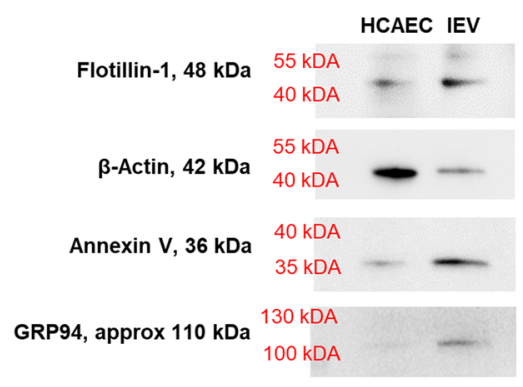

HCAEC IEV

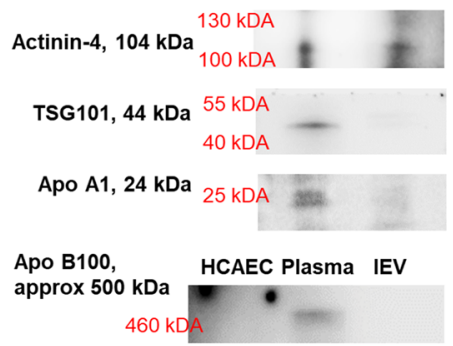

(H)

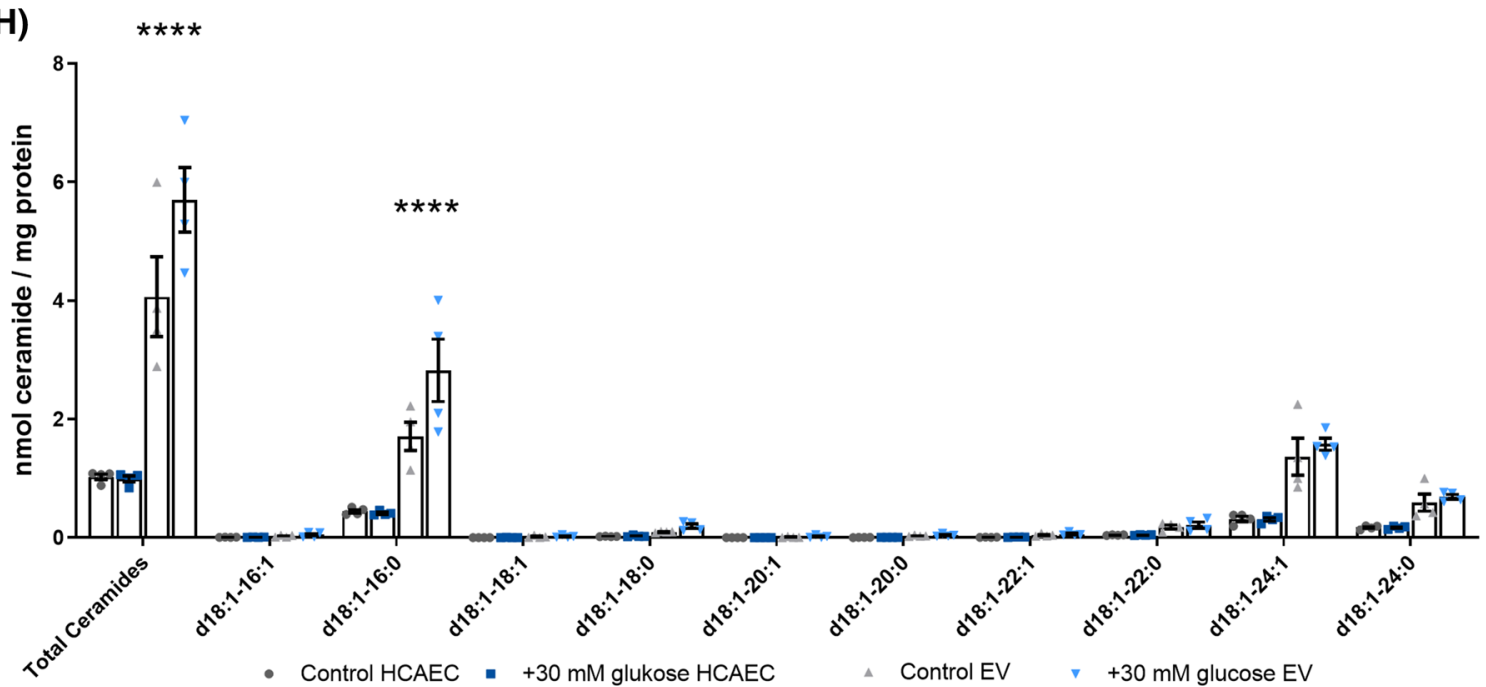


४Fig. 1 A Schematic diagram of the IEV isolation protocol. B Flowcytometric analysis of apoptosis induction in HCAECs after hyperglycemic injury, $n=6$, with representative dot blots. C MTT viability assay in HCAECs after hyperglycemic injury, $n=6$. $\mathbf{D}+\mathbf{E}$ Distribution of $1 \mathrm{EV}$ size and relative $1 \mathrm{EV}$ s released after hyperglycemic injury in HCAECs, as analyzed by nanoparticle tracking analysis, $n=3$. $\mathbf{F}$ Direct electron microscopic imaging of HCAEC-derived IEVs with negative staining, representative image. G Characterization of IEVs by immunoblotting for Flotillin-1, $\beta$-Actin, Annexin V, GRP94, Actinin-4, TSG101, Apolipoprotein A1, Apolipoprotein B100 (with additional human plasma as an antibody control). H Mass spectrometric analysis of ceramides in HCAECs and lEVs after hyperglycemic injury. All data are presented as individual experiments with the mean $\pm \mathrm{SEM} ; n . s$. not significant, $* p<0.05$, $* * * p<0.001$, $* * * * p<0.0001$. ANOVA + Bonferoni's multiple comparison test were used for $\mathbf{B}+\mathbf{C}$, unpaired $t$-Test for $\mathbf{E}$, two-way ANOVA + Bonferoni's multiple comparison test were used for $\mathbf{H}$

FACSCanto II (BD Bioscience) machine, another $600 \mu \mathrm{l}$ of Annexin $\mathrm{V}$ binding buffer were added. For compensation, gating, and quantification we used the software FlowJo V10 (BD Bioscience), as previously reported [42]. A representative gating strategy is displayed in Figure S1.

\section{Nanoparticle tracking analysis}

For nanoparticle tracking analysis, we used a ZetaView BASIC NTA-Nanoparticle Tracking Video Microscope PMX-120 (Particle Metrix). The analysis were performed as previously described [32]. For the analysis of lEVs from murine plasma, the $\mathrm{lEVs}$ from $3 \mu \mathrm{l}$ plasma were diluted in $1000 \mu \mathrm{PBS}$ and analyzed by NTA. A concentration row confirmed, that the resulting concentrations were in the linear range of the NTA.

\section{Viability assay (MTT)}

HCAEC viability was assessed by use of the MTT Cell Growth Assay Kit (Sigma-Aldrich, Cat\# CT02). The assay was carried out in 96-well plates, according to the manufacturer's recommendations. In brief, $10 \mu \mathrm{l}$ of the AB Solution (MTT) were added to each well containing $100 \mu \mathrm{l}$ of medium with the respective stimulant. The cells were incubated at $37^{\circ} \mathrm{C}$ for $4 \mathrm{~h}$. Subsequently, $100 \mu \mathrm{l}$ isopropanol with $0.04 \mathrm{~N}$ $\mathrm{HCl}$ were added and the formazan was dissolved. Finally, absorbance was measured in a Tecan Infinite M200 Plate Reader (Tecan), using a test wavelength of $570 \mathrm{~nm}$ and a reference wavelength of $630 \mathrm{~nm}$.

\section{Lipid extraction}

Lipid isolation was performed as previously published [49]. In brief, approximately $1.0 \times 10^{7}$ HCAECs and their respective lEVs were harvested with $1 \mathrm{ml}$ (HCAECs) or $0.5 \mathrm{ml}$ (lEVs) of a hypo-osmolar buffer containing $10 \mathrm{mM}$ HEPES and $0.5 \mathrm{mM}$ EDTA, together with Roche Protease
Inhibitor Cocktail (Roche, Cat\# 4693132001), and sonicated for $15 \mathrm{~min}$ in ice-cold water. The protein concentration was assessed with a Qubit-4 Fluorometer (Thermo Fisher Scientific) using the Qubit ${ }^{\mathrm{TM}}$ Protein Assay Kit (Thermo Fisher Scientific, Cat\# Q33211), according to the manufacturer's instructions. Lipid extraction was performed with a homogenous phase of chloroform and methanol $(1: 2, v / v)$, overnight at $37^{\circ} \mathrm{C}$. Subsequently, the sample was centrifuged at $2500 \mathrm{rpm}$ for $15 \mathrm{~min}$. The supernatant was transferred to a new container and dried under $N_{2}$ flow. The extraction was then repeated with chloroform/methanol $(1: 1, v / v)$ and with chloroform/methanol $(2: 1, v / v)$, with a 1-hour incubation at $37{ }^{\circ} \mathrm{C}$ for each step. For the first analysis (glucose treatment) the procedure was followed by alkaline hydrolysis as previously described [49]. For the other experiments (addition of $\mathrm{C} 16$ ceramide or GW4869), the lipids were extracted by use of silica columns (Strata-1 Silica, $55 \mu \mathrm{m}, 70 \AA, 100 \mathrm{mg}$; Phenomenex, Aschaffenburg, Germany). To this end, the lipids were dissolved in chloroform and loaded onto the columns. The columns were washed three times with chloroform and ceramides were eluted with acetone/isopropanol $(1: 1, v / v)[50]$.

\section{Quantification of sphingolipids using mass spectrometry}

Sphingolipid analysis was carried out using a QTRAP 6500 + LC-MS/MS system (Sciex, Darmstadt), as previously described [51].

\section{Immunoblotting}

For immunoblotting of IEVs and HCAECs, the samples were lysed in RIPA buffer (Sigma-Aldrich, Cat\# R0278) with 1:25 Protease Inhibitor Cocktail (Roche, Cat\# 4693132001). After ultra-sonication for $10 \mathrm{~min}$, the protein concentration was assessed in a Qubit-4 Fluorometer (Thermo Fisher Scientific) with the Qubit ${ }^{\mathrm{TM}}$ Protein Assay Kit (Thermo Fisher Scientific, Cat\# Q33211). The assay was carried out according to the manufacturer's instructions. $50 \mu \mathrm{g}$ total protein ( $10 \mu \mathrm{g}$ were used for the analysis of cellular lysate in Fig. 2C) from human or murine samples were diluted 2:1 in $3 \times$ Laemmli buffer and loaded onto an SDS-PAGE gel (Biorad, Cat\#456-1084 and 456-1024) using the Mini PROTEAN System (Biorad). For western blotting, we used a RotiNC nitrocellulose membrane (Carl Roth GmbH, HP40.1). Subsequently, the membranes were blocked with 5\% BSA (Sigma-Aldrich) for one hour at RT. As primary antibodies for human lEVs, we used monoclonal mouse anti-Annexin $\mathrm{V}$ antibody (Abcam, Cat\# ab54775) 1:1000, mouse anti-Flotillin-1 antibody (BD Bioscience, Cat\# 610820) 1:1000, mouse anti-GRP94 antibody (Abcam, Cat\# ab210960) 1:500, recombinant rabbit monoclonal anti-TSG101 antibody 


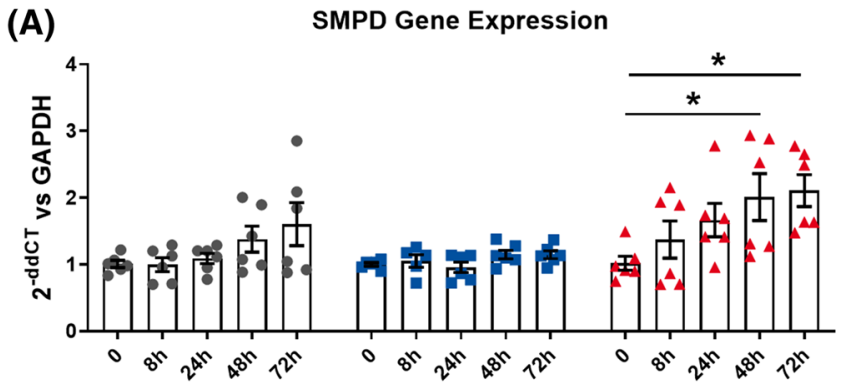

Duration of hyperglycemic injury

- SMPD1 - SMPD2 a SMPD3

(C)

Immunoblotting of nSMase2 after hyperglycemic inury

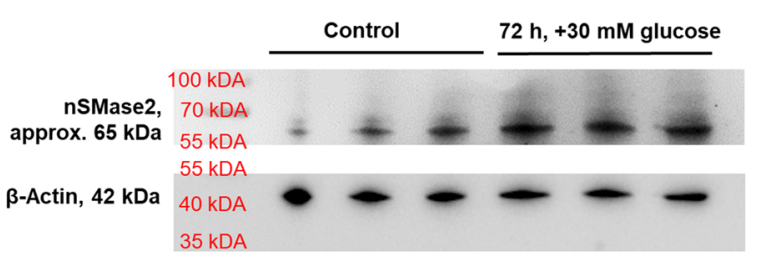

(B) SMase activity assay

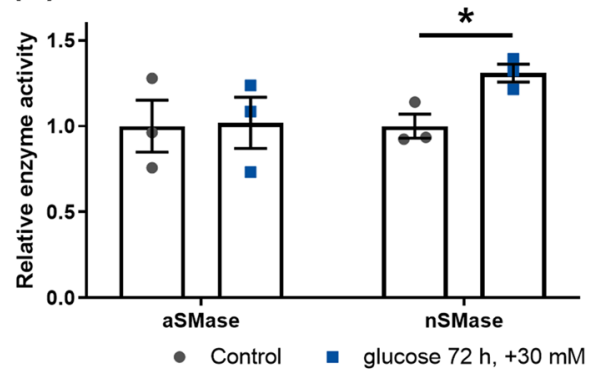

(D)

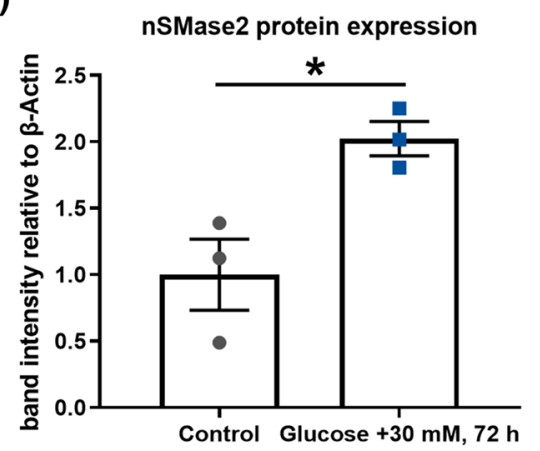

(E)

MTT viability assay

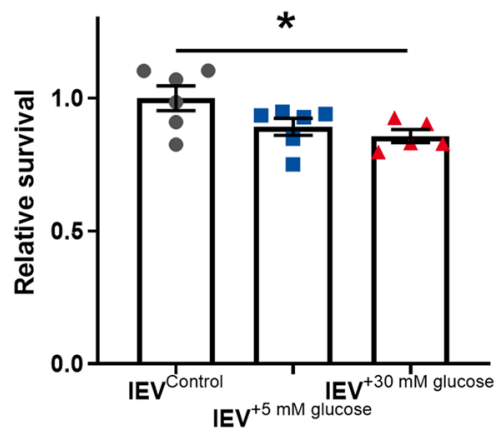

(G)

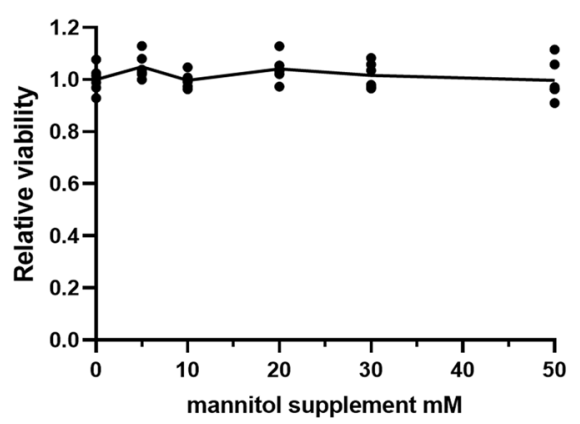

(F)

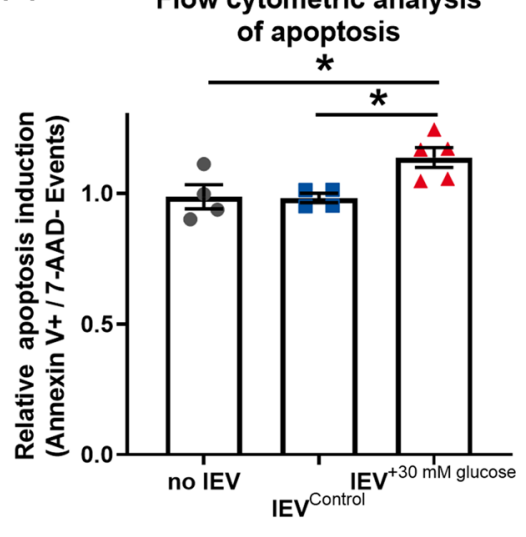

(H)

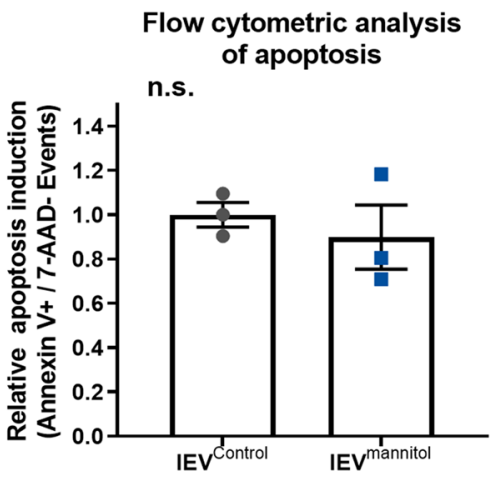

No IEV

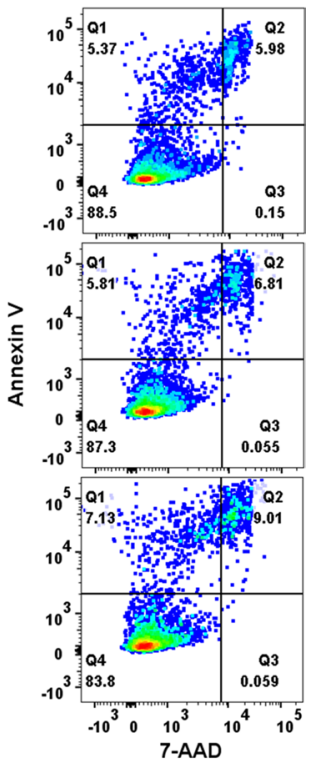

IEVControl

$+30 \mathrm{mM}$ IEVglucose 
4Fig. 2 A Timeline of gene expression of SMPD1-3 in HCAECs after hyperglycemic injury, presented as $2^{-\mathrm{ddCT}}$ vs $G A P D H, n=6$. B Acidic and neutral SMase enzyme activity in HCAECs after $72 \mathrm{~h}$ of hyperglycemic injury, $n=5-6$. $\mathbf{C}+\mathbf{D}$ Immunoblotting for nSMase 2 and $\beta$-Actin after $72 \mathrm{~h}$ of hyperglycemic injury, with quantification, $n=3$. E MTT viability assay in HCAECs that were treated with $1 E V$ s from HCAECs after hyperglycemic injury, $n=6$. F Flow-cytometric analysis of the induction of apoptosis in HCAECs that were treated with IEVs from HCAECs after hyperglycemic injury, (right) representative dot blots, $n=5$. G MTT viability assay in HCAECs after treatment with mannitol, $n=6$. H Flow-cytometric analysis of the induction of apoptosis in HCAECs that were treated with lEVs from HCAECs after osmotic injury with mannitol, $n=3$. All data are presented as individual experiments with the mean $\pm \mathrm{SEM} ;$ n.s. not significant, ${ }^{*} p<0.05$, ANOVA + Bonferoni's multiple comparison test were used for $\mathbf{A}, \mathbf{E}, \mathbf{G}$, and unpaired $t$-Test for $\mathbf{B}, \mathbf{D}, \mathbf{F}, \mathbf{H}$

(Abcam Cat\# ab125011) 1:500, anti-alpha Actinin-4 antibody (Abcam Cat\# ab108198) 1:500, mouse anti-Apolipoprotein B Monoclonal Antibody (Thermo Fisher Scientific, Cat\# MA5-14671) 1:500, Chicken anti-ApoA1 polyclonal Antibody (Invitrogen, Cat\# PA5-20721) 1:500, mouse anti$\beta$-Actin antibody (Sigma-Aldrich, Cat\# A1978) 1:2000, in BSA $5 \%$ overnight at $4{ }^{\circ} \mathrm{C}$. For sEVs, we used anti-CD81 Monoclonal Antibody (Thermo Fisher Scientific, Cat\# 10630D) 1:500, and anti-Alix Mouse mAb (Cell Signaling Technology, Cat\# 2171S) 1:1000. For murine lEVs, we used rabbit monoclonal anti-Flotillin-1 antibody (Abcam Cat\# ab41927) 1:1000 and rabbit polyclonal anti-Histone H3 antibody (Abcam Cat\# ab1791) 1:1000, under identical conditions. For the cellular analysis of nSMase 2 expression, a rabbit polyclonal anti-SMPD3 antibody (Thermo Fisher Scientific, Cat\# PA5-49140) 1:1000 as well as the abovementioned anti- $\beta$-Actin antibody were used. The membranes were washed three times with $0.1 \%$ TBST and incubated with an HRP-conjugated monoclonal rat anti-mouse-IgG antibody (Sigma-Aldrich, Cat\# A9044) 1:3000 or with an HRP-conjugated polyclonal goat anti-rabbit-IgG (Carl Roth, Cat\# 4750.1) 1:1000 or with a goat anti-chicken IgG HRPconjugated antibody (Abcam, Cat\# ab97135) 1:3000 in 5\% BSA for one hour at RT. Thereafter, the membranes were washed again two times with $0.1 \%$ TBST and once with PBS. For development, the ECL primer western blotting detection reagent (Sigma-Aldrich) was used. Image acquisition was performed by use of a ChemiDoc Imaging System (Biorad, Cat\# 17001401).

\section{SiRNA transfection}

For siRNA transfection of HCAECs, SMPD3 siRNA (Invitrogen, Cat\# AM16708, Assay ID 132606) as well as silencer negative control No. 1 siRNA (Invitrogen, Cat\# AM4611) were used at a final concentration of $15 \mathrm{nM}$ together with Lipofectamine RNAiMAX Transfection reagent (Invitrogen,
Cat\# 13778150) at a final concentration of $3.75 \mu \mathrm{l} / \mathrm{ml}$ for $48 \mathrm{~h}$.

\section{Electron microscopy of IEVs}

For transmission electron microscopic imaging, lEVs were harvested from one $10-\mathrm{cm}$ dish $\left(60 \mathrm{~cm}^{2}\right.$ growth area), as described above. After isolation, the lEVs were resuspended in $10 \mu \mathrm{l}$ PBS with Protease Inhibitor Cocktail (Roche, Basel, Switzerland, Cat\# 4,693,132,001). $5 \mu 1$ of the sample were loaded on Formvar-coated copper grids (Science Services, München, Germany) and fixed twice with $2 \%$ paraformaldehyde for $5 \mathrm{~min}$ and subsequently for $5 \mathrm{~min}$ with $1 \%$ glutaraldehyde. After extensive washing with distilled water, the sample was contrasted with $1.5 \%$ uranyl acetate for 4 min. Image acquisition was performed with a Jem-2100Plus (Jeol), which was operated at $200 \mathrm{kV}$, together with a Gatan OneView $4 \mathrm{~K}$ camera.

\section{RNA isolation and quantitative real-time PCR}

RNA was isolated with Trizol (Invitrogen, Cat\# 15596026) and chloroform, as previously described [47]. In brief, the cells were lysed in Trizol, after washing with ice-cold PBS. RNA was isolated with chloroform, precipitated with isopropanol, washed twice with ethanol, dried, and resuspended in water. The quality and concentration of the RNA were assessed using a Nanodrop2000 (Thermo Fisher Scientific).

For reverse transcription, we used $0.5 \mu \mathrm{g}$ RNA and the Omniscript RT Kit (Qiagen, Cat\# 205111) with a "random" primer (Roche, Cat\# 11034731001), following the manufacturer's protocol. For real-time PCR, we diluted $20 \mu \mathrm{l}$ cDNA preparation with $480 \mu \mathrm{l}$ water and used $9 \mu \mathrm{l}$ of the dilution together with $11 \mu \mathrm{l}$ of the Taqman Gene Expression Master Mix (Applied Biosystems, Cat\# 4440040), and the respective Taqman probes for SMPD1, SMPD2, SMPD3, CERS5, CERS6, and GAPDH (all from Thermo Fisher Scientific: SMPD1 Cat\# 4331182, Assay ID Hs04183448_m1; SMPD2, Cat\# 4331182, Assay ID Hs00162006_m1; SMPD3, Cat\# 4331182, Assay ID Hs00920354_m1; CerS5, Cat\# 4331182, Assay ID Hs00332291_m1; CerS6, Cat\# 4331182, Assay ID Hs00826756_m1; GAPDH, Cat\# 4331182, Assay ID Hs02786624_g1) in a 7500 HT real-time PCR instrument (Applied Biosystems). We used GAPDH as an internal control and relative expression was calculated as $2^{-\Delta \Delta C T}$.

\section{Sphingomyelinase activity assays}

For the assessment of nSMase activity, a Sphingomyelinase Assay Kit (Abcam Cat\# ab138876) was used according to the manufacturer's instructions. In brief, cellular lysates from HCAECs were prepared using Mammalian Cell Lysis Buffer (Abcam Cat\# ab179835). The protein concentration 
(A) IEV Isolation protocol

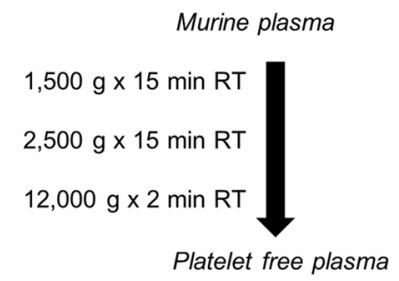

$20,000 \mathrm{~g} \times 40 \mathrm{~min} 4^{\circ} \mathrm{C}$

Washing with PBS

$20000 \mathrm{~g} \times 40 \mathrm{~min} 4^{\circ} \mathrm{C}$

Immediate use of EV pellet for experiments
(B)

Streptozotocin (150 mg / kg BW i.p.)

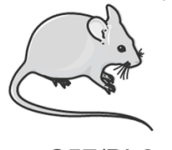

C57/BL6-J

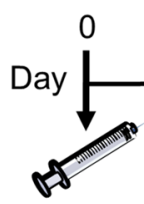

(C)

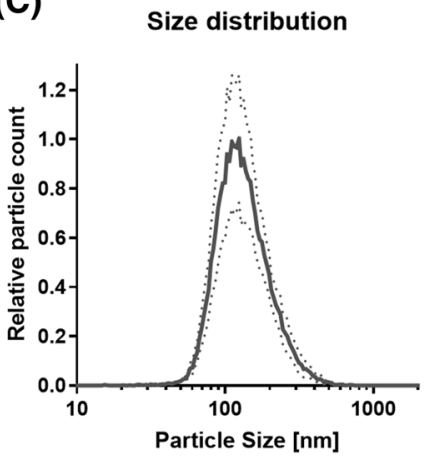

(D)

Durine IEV characterization by Immunoblotting

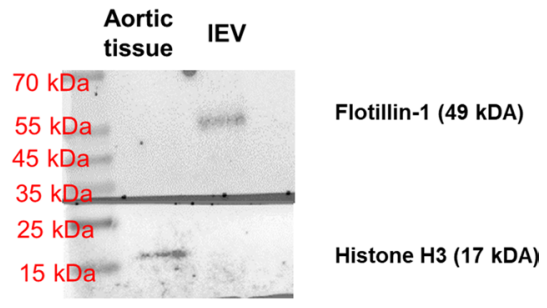

(E)

\begin{tabular}{lcc}
$\begin{array}{l}\text { Blood } \\
\text { glucose } \\
\text { level } \\
\text { [mmol/L] }\end{array}$ & d 0 & d 21 \\
\hline Control 1 & 9.0 & 8.8 \\
Control 2 & 10.6 & 10.6 \\
Control 3 & 7.7 & 7.7 \\
Control 4 & 8.7 & 8.6 \\
Control 5 & 8.1 & 9.4 \\
STZ 1 & 7.4 & $>44.4$ \\
STZ 2 & 8.4 & $>44.4$ \\
STZ 3 & 7.2 & 30.3 \\
STZ 4 & 7.1 & $>44.4$ \\
STZ 5 & 9.4 & 29.6
\end{tabular}

(G)

Murine plasma IEV levels after $21 \mathrm{~d}$ of STZ treatment

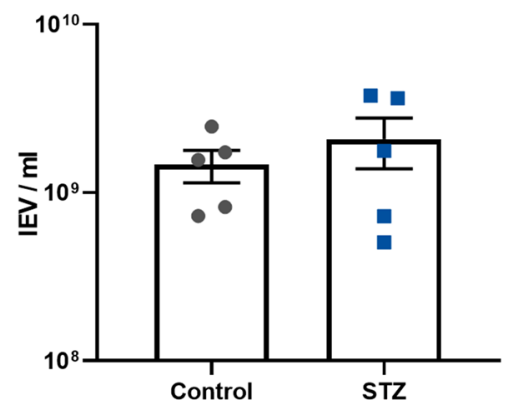

(F)

Flow cytometric analysis of apoptosis in MCAECs
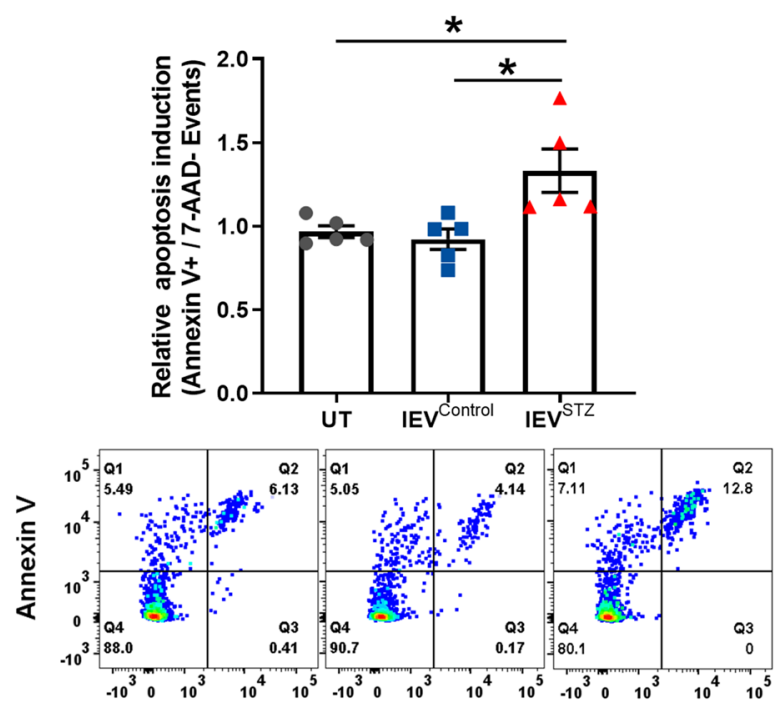

7-AAD 
4 Fig. 3 A Schematic diagram of the IEV isolation protocol from murine blood. B Diagram of the experimental set-up of the mouse experiment to induce hyperglycemia through streptozotocin injection. C Analysis of murine $1 \mathrm{EV}$ size distribution by nanoparticle tracking analysis, $n=3$. D Characterization of $1 \mathrm{EVs}$ by immunoblotting for Histone H3 and Flotillin-1. E Table of individual blood sugar levels of the mice. F Flow-cytometric analysis of the induction of apoptosis in MCAECs that were treated with $1 E V s$ from hyperglycemic and normoglycemic mice, $n=5$. G Nanoparticle tracking analysis of $1 \mathrm{EV}$ concentration in murine plasma from control animals and STZtreated animals, $n=5$. * $p<0.05$, ANOVA+Bonferoni's multiple comparison test was used for $\mathbf{E}$

of the lysates was assessed as described above. $5 \mathrm{mg}$ of total protein in $50 \mu \mathrm{l}$ were used for the assay together with SMase reaction buffer and sphingomyelin (both part of the kit) for 2 hours at $37{ }^{\circ} \mathrm{C}$ to allow for hydrolysis of sphingomyelin. Phosphocholine production was then quantified with the AbBlue Indicator system, by measuring the absorbance of the samples at $655 \mathrm{~nm}$ in a Tecan Infinite M200 Plate Reader (Tecan). Sphingomyelinase activity was calculated by use of a standard, which was provided with the kit.

For the assessment of acidic sphingomylinase activity, the Acidic Sphingomyelinase Assay Kit (Abcam, ab190554) was used according to the manufacturer's instructions. Like for the nSMase assay, HCAECs were lysed in Mammalian Cell Lysis Buffer (Abcam, Cat\# ab179835) and the protein concentration was assessed. For the aSMase assay, the protein concentration was adjusted to $80 \mu \mathrm{g} / \mu \mathrm{l}$ and $50 \mu \mathrm{l}$ were used for incubation with the respective buffers for 2 hours at $37{ }^{\circ} \mathrm{C}$ to allow hydrolysis of the sphingomyelin. Phosphocholine production was then quantified with the AbRed Indicator system by measuring the fluorescence signal (Ex/ $\mathrm{Em}=540 / 590 \mathrm{~nm}$ ) in a Tecan Infinite M200 Plate Reader (Tecan). ASMase enzyme activity was calculated as relative activity normalized to the untreated sample.

\section{Caspase $3 / 7$ assay}

Caspase 3/7 activity was assessed by use of the CellEvent Caspase-3/7 Green Detection Reagent (Thermo Fisher Scientific, Cat \# C10423) in a 96-well plate after stimulation with $5.0 \times 10^{8} / \mathrm{ml} \mathrm{lEVs}$ for $24 \mathrm{~h}$. The assay was then carried out as recommended by the manufacturer, and the plates were imaged with a Zeiss Axio Observer microscope and analyzed with the ZEN 2.3 pro software.

\section{Apoptosis proteome profiler array}

The Proteome Profiler Human Apoptosis Array Kit (R\&D Systems Cat\#ARY009) was used according to the manufacturer's instructions. We used $300 \mu \mathrm{g}$ total protein lysate from HCAECs after stimulation with $5.0 \times 10^{8} / \mathrm{ml} \mathrm{lEVs}$ for $24 \mathrm{~h}$. Image acquisition was performed by use of a ChemiDoc Imaging System (Biorad, Cat\# 17001401).

\section{Animals}

All animal experiments were carried out in accordance with the animal protection law stated in the German civil code. All investigations were approved by the National Office for Nature, Environment and Consumer Protection in Recklinghausen, Nordrhein-Westfalen. All procedures conformed to the guidelines from Directive 2010/63/EU of the European Parliament on the protection of animals used for scientific purposes. We used $n=10,10$-week-old male C57BL6-J mice, which were purchased from Janvier Labs. To induce hyperglycemia, we used a standard streptozotocin (STZ) injection protocol from the Diabetic Complications Consortium [52]. The mice were randomly divided into two groups of which the first was injected with $150 \mathrm{mg} / \mathrm{kg}$ bodyweight of STZ in a $29.4 \mathrm{mg} / \mathrm{ml}$ sodium citrate buffer after $6 \mathrm{~h}$ of starvation or with sodium citrate buffer only (control animals). Before the injection, blood sugar levels were confirmed to be in the normal range, with an average of $8.3 \mathrm{mmol} / \mathrm{L}$, as previously reported for C57BL6-J mice [53]. After 21 days, the blood sugar was assessed again to confirm hyperglycemia and the mice were euthanized by cervical dislocation. Blood was immediately drawn from the inferior caval vein into a sodium-citrate-containing syringe to give a final concentration of $3.2 \%$ citrate. The blood was then centrifuged in an Eppendorf Centrifuge 5430 with a Rotor FA-45-24-11-HS to remove blood cells and platelets: $15 \mathrm{~min}$ at $1500 \times g$ at RT, $15 \mathrm{~min}$ at $2000 \times g$ at RT, and subsequently for $2 \mathrm{~min}$ at $13,000 \times g$ at RT. The platelet-free plasma was then snap frozen in liquid nitrogen and stored at $-80^{\circ} \mathrm{C}$. $1 \mathrm{EVs}$ were isolated from $250 \mu \mathrm{l}$ of platelet-free plasma by centrifuging twice at $20,000 \times g$ for $40 \mathrm{~min}$. The $1 \mathrm{EV}$ s were then resuspended in $250 \mu \mathrm{l}$ complete-mouse endothelialcell medium to achieve the same concentrations of lEVs as in the murine blood. The IEV suspension was then incubated with MCAECs in a 24-well plate at $90 \%$ confluence. After $24 \mathrm{~h}$, apoptosis of the MCAECs was assessed using a FITC Annexin V Apoptosis Detection Kit with 7-AAD, as described above, the only adaptation being the use of $2 \mu \mathrm{l}$ Annexin V staining solution and $1 \mu \mathrm{l}$ 7-AAD staining solution.

\section{NBD-C12 transfer experiment}

To assess the transfer of ceramides by $1 E V s$ among HCAECs, we used NBD-C12-ceramide (NBD-C12, Avanti Polar Lipids, Cat\# 810211P-1mg). NBD-C12 ceramide was dissolved in DMSO at a concentration of $5 \mathrm{mM}$ and incubated with HCAECs at concentrations of 10 or $20 \mu \mathrm{M}$, or the respective amounts of DMSO were added as a control, for $24 \mathrm{~h}$ to allow for uptake. To control the effective uptake, the cells were washed three times with warm PBS to remove excess NBD-C12, fixed with $4 \%$ PFA for $30 \mathrm{~min}$ 


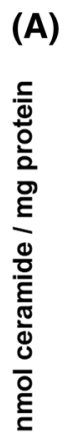

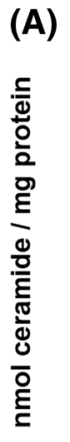
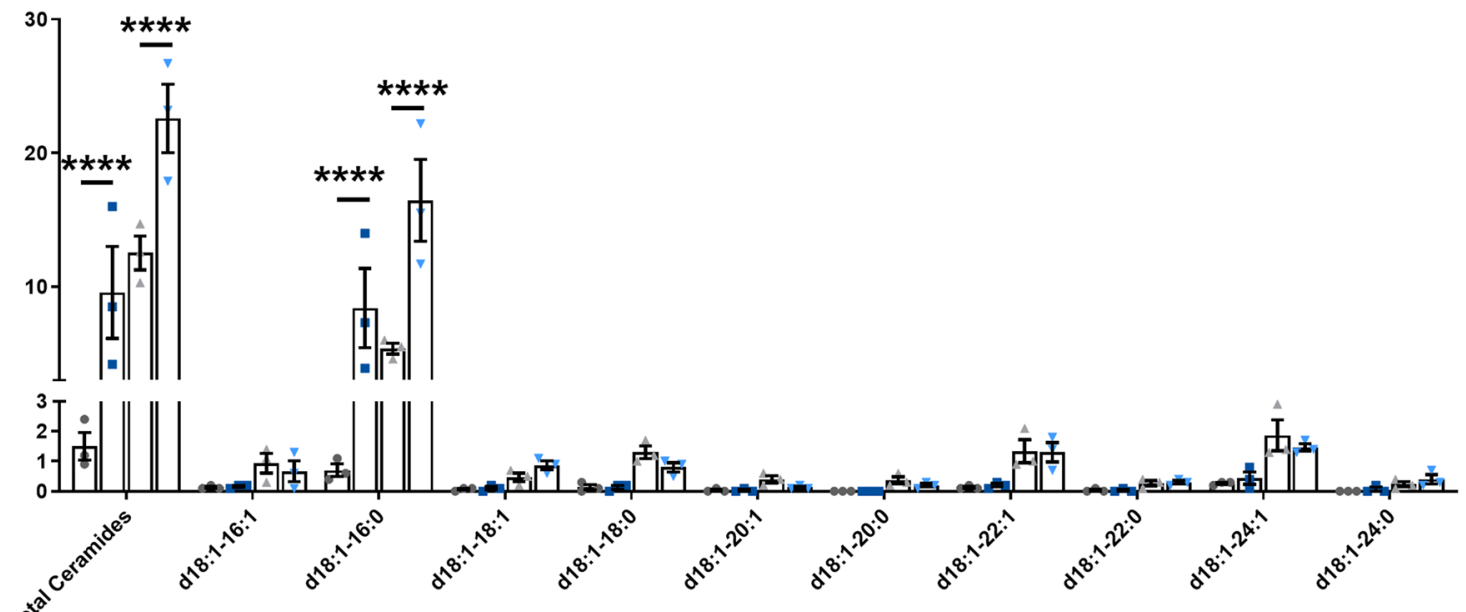

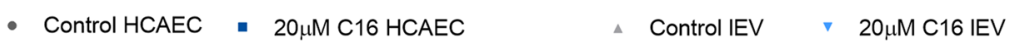

(B)

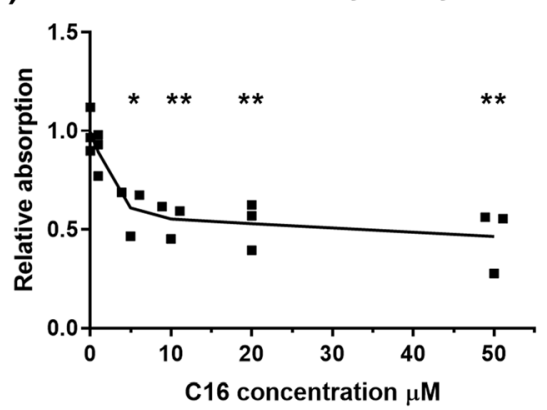

(D)

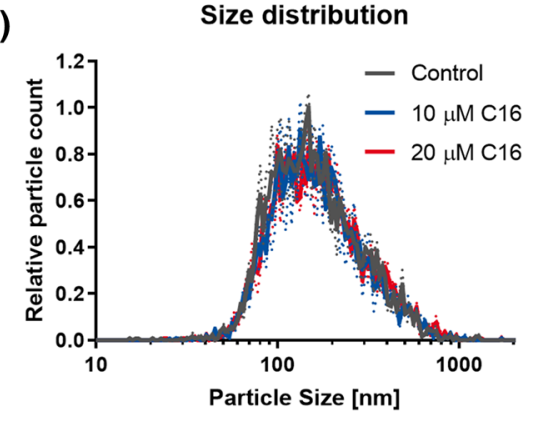

(F)

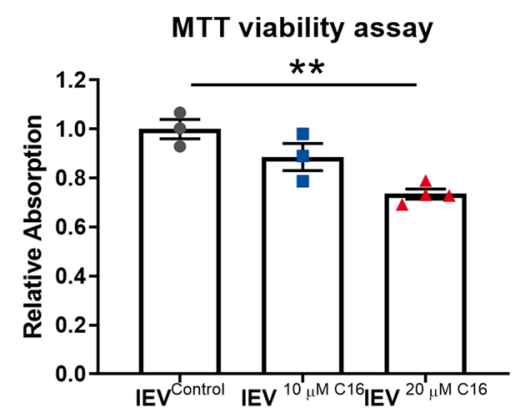

(C) Flow cytometric analysis of apoptosis

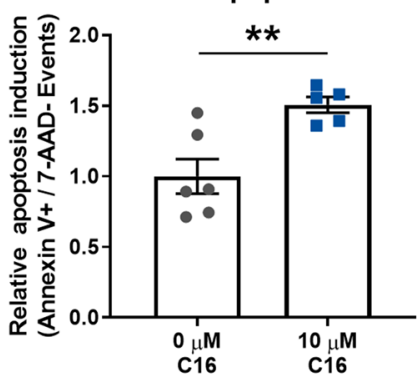

IEV release

(E)

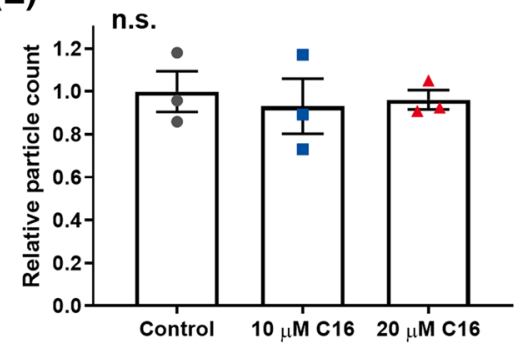

(G) Flow cytometric analysis of
apoptosis

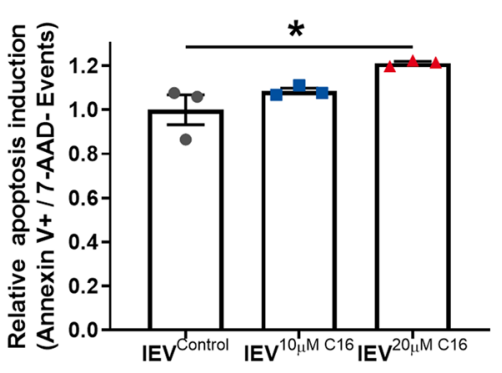

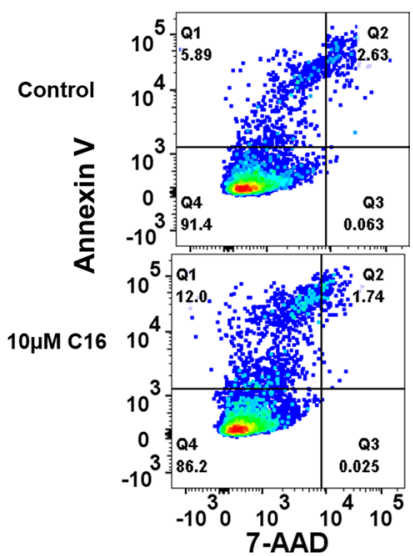

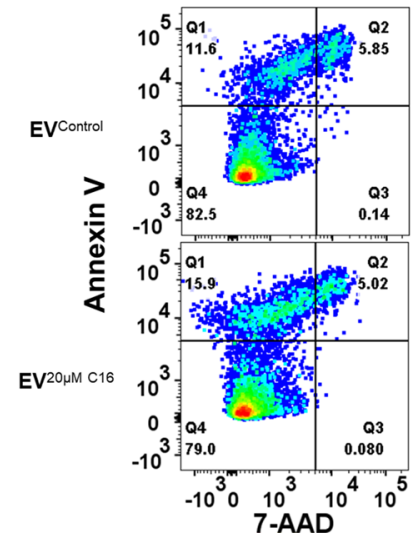


4Fig. 4 A Mass spectrometric analysis of ceramides in HCAECs and IEVs after treatment with $20 \mu \mathrm{M} \mathrm{C} 16$ ceramide for $24 \mathrm{~h}, n=3$. B MTT viability assay in HCAECs after treatment with $\mathrm{C} 16$ ceramide for $24 \mathrm{~h}, n=6$. C Flow-cytometric analysis of the induction of apoptosis in HCAECs after treatment with C16 ceramide for $24 \mathrm{~h}$, (right) representative dot blots, $n=6$. $\mathbf{D}+\mathbf{E}$ Distribution of $1 \mathrm{EV}$ sizes and relative $1 \mathrm{EV}$ release after treatment of HCAECs with C16 ceramide for $24 \mathrm{~h}$, as analyzed by nanoparticle tracking analysis, $n=3$. F MTT viability assay in HCAECs after treatment with $1 \mathrm{EV}$ s from HCAECs after C16 ceramide treatment, $n=3-4$. G Flow-cytometric analysis of apoptosis induction in HCAECs after treatment with lEVs from HCAECs after C16 ceramide treatment with representative dot blots, $n=3$. All data are presented as individual experiments with the mean \pm SEM; n.s. not significant, ${ }^{*} p<0.05$, $* * p<0.01$, $* * * * p<0.0001$. An unpaired $t$-test was used for $\mathbf{C}$; ANOVA+Bonferoni's multiple comparison test were used for $\mathbf{B}, \mathbf{E}-\mathbf{G}$; two-way ANOVA + Bonferoni's multiple comparison test were used for A

at $4{ }^{\circ} \mathrm{C}$ and counterstained with VECTASHIELD mounting medium with DAPI (Vector Laboratories, Cat\# H-1200). For the transfer experiment, HCAECs were treated with NBD-C12 ceramide at 10 or $20 \mu \mathrm{M}$ for $24 \mathrm{~h}$. After extensive washing to remove excess NBD-C12, the cells were incubated for $24 \mathrm{~h}$ with serum-free medium to trigger lEV release. From the medium, lEVs were isolated as described above. Additionally, the spontaneous formation of NBD-C12 ceramide micelles, which could be co-isolated with lEVs, was investigated. To this end, $2 \mathrm{ml}$ of a $20 \mu \mathrm{M}$ NBD-C12 solution in PBS were subjected to the same centrifugation steps as for lEV isolation. The isolated lEVs or "pseudoEVs" from NBD-C12 PBS were incubated with native HCAECs for $12 \mathrm{~h}$ to allow for uptake. Subsequently, the cells were washed three times extensively with PBS, fixed with $4 \%$ PFA for $30 \mathrm{~min}$ at $4{ }^{\circ} \mathrm{C}$, and counterstained with VECTASHIELD mounting medium with DAPI (Vector Laboratories, Cat\# H-1200). Images were acquired by use of a Zeiss Axio Observer microscope and analyzed with the ZEN 2.3 pro software.

\section{Quantification and statistical analysis}

Data are presented as the mean \pm standard error of the mean (SEM) throughout the manuscript. The number of independent replications of the respective experiment is reported as a number, $n$, in the figure legends. All statistical analyses were performed with the software Prism8. Statistical details are displayed in the figure legends. Means of two groups were compared with an unpaired $t$-test. Means of more than two groups were compared by a one-way ANOVA and Bonferoni's post hoc test. To account for multiple testing, a twoway ANOVA and Bonferoni's post hoc test were applied for mass spectrometric analyses of sphingolipids. All reported $\mathrm{p}$ values are two-sided.

\section{Results}

\section{Hyperglycemic injury induces endothelial-cell apoptosis and enhances ceramide export into large extracellular vesicles}

To simulate diabetic conditions in vitro, HCAECs were incubated with regular medium that was supplemented with 5 or $30 \mathrm{mM}$ glucose. While adding $5 \mathrm{mM}$ glucose did not have any effect on cell viability or the induction of apoptosis, the addition of $30 \mathrm{mM}$ glucose for $72 \mathrm{~h}$ led to a decline of HCAEC viability and an induction of apoptosis (Fig. 1B +C). After $24 \mathrm{~h}$ of serum-free culture, lEVs were isolated from the HCAECs and characterized according to the recommendations of the International Society for Extracellular Vesicles (ISEV) [54]. As shown by nanoparticle tracking analysis, high concentrations of glucose did not significantly change the release or size distribution of $\mathrm{lEVs}$ (Fig. 1D+E). Electron microscopic imaging showed typical, cup-shaped lEVs mainly 100-200 nm in size and immunoblotting confirmed the presence of Annexin V, Flotillin-1, Actinin-4, and GRP94 as markers for EVs. (Fig. 1F+G). The cytoplasmic protein $\beta$-Actin, the ESCRT member TSG101, which is enriched in small EVs, as well as the Apolipoproteins A1 and B100 were only present in traces. To evaluate the effect of hyperglycemic injury on vesicular ceramide export, we performed a mass spectrometric analysis of ceramides from IEVs and HCAECs after hyperglycemic injury (Fig. 1H). We found that injury with an addition of $30 \mathrm{mM}$ glucose for $72 \mathrm{~h}$ led to an increase of the total ceramide levels in IEVs. The increase in IEV ceramide levels was driven by a significant increase in $\mathrm{d} 18: 1 / 16: 0$ ceramide (C16), which was also the most abundant ceramide in nonglucose-treated HCAEC-derived IEVs. Interestingly, cellular ceramide levels were not changed significantly after glucose injury (Fig. 1H). Besides ceramides, we also found dihydroceramides to be significantly increased in $\mathrm{IEVs}$, however at much lower levels. Sphingomyelin levels in IEVs were not significantly changed in contrast to cellular sphingomyelins, which were significantly reduced after hyperglycemic injury (Figure S2).

\section{Glucose injury triggers SMPD3 activation and leads to increased apoptosis in IEV recipient cells}

As sphingomyelinases have been shown to be involved in EV release [30], we evaluated the effect of hyperglycemic injury on SMPD1-3 expression in a time-course experiment. We found that SMPD3 RNA expression was significantly upregulated after $48 \mathrm{~h}$ and $72 \mathrm{~h}$ of incubation with $+30 \mathrm{mM}$ glucose, while expression of $S M P D 1$ and SMPD2 RNA were unchanged (Fig. 2A). The increased expression of SMPD3 
(A)
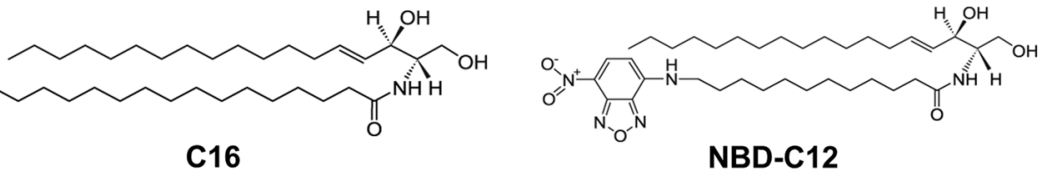

(B) EV releasing cells
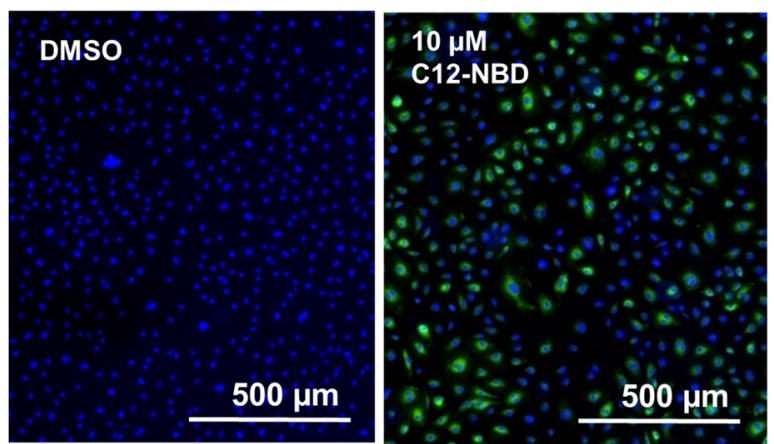

DAPI
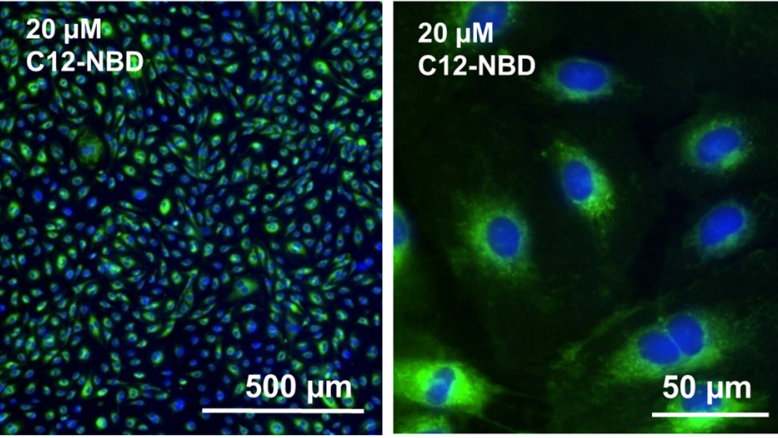

NBD-C12

(C) EV recipient cells
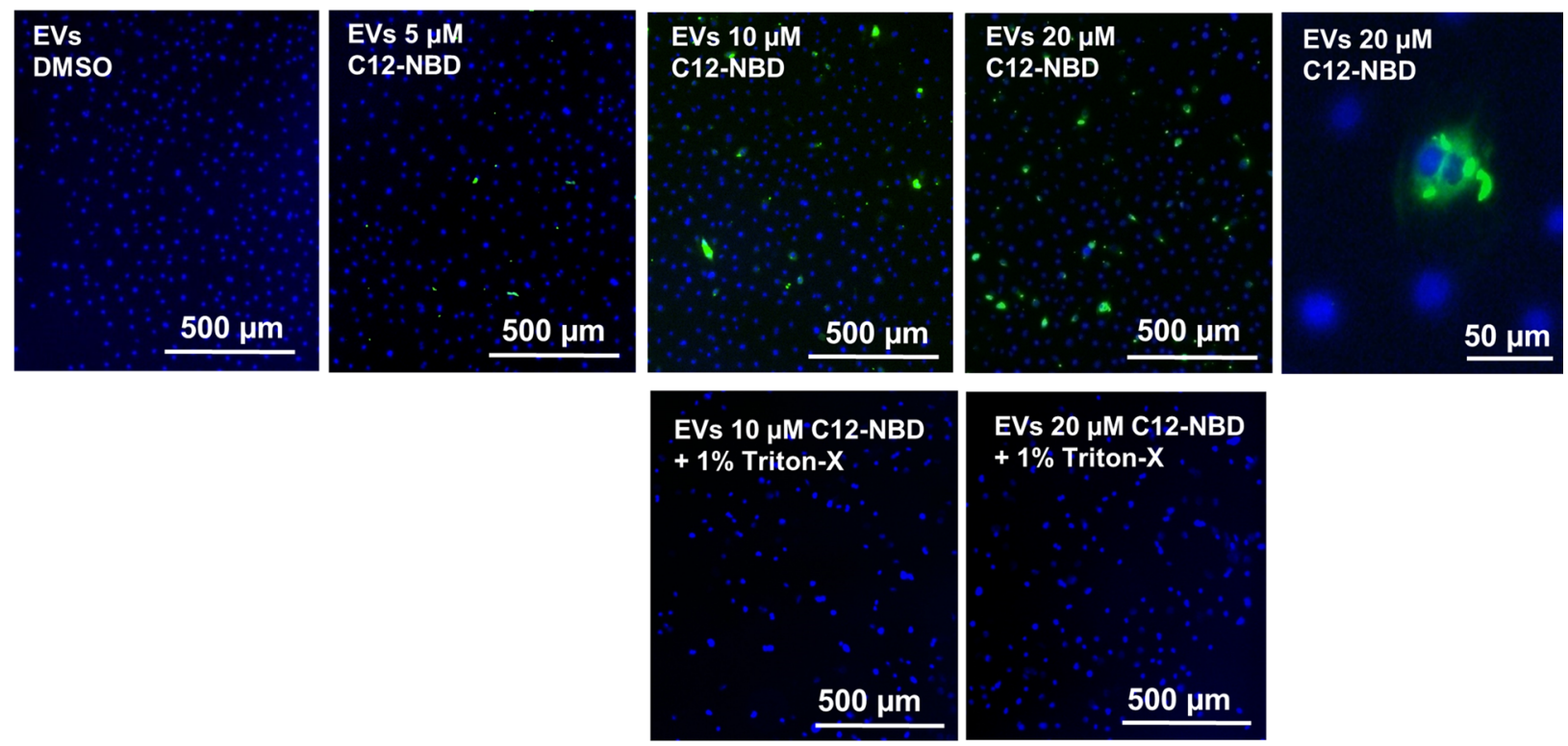

\section{EVs $20 \mu \mathrm{M}$ C12-NBD}

$+1 \%$ Triton-X

(D)

Absolute EV uptake

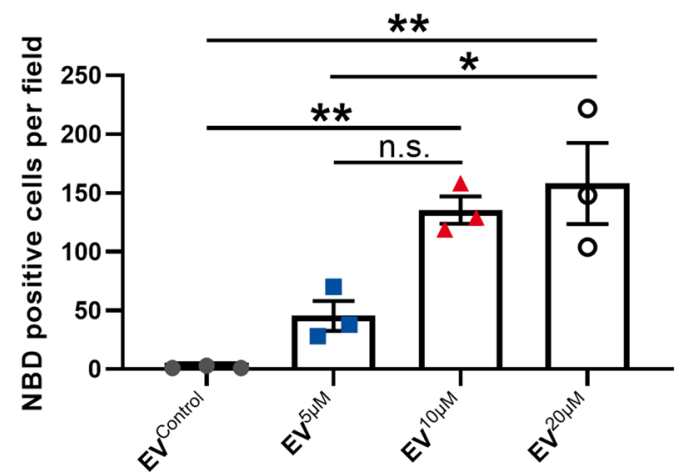

(E)

Reative EV uptake

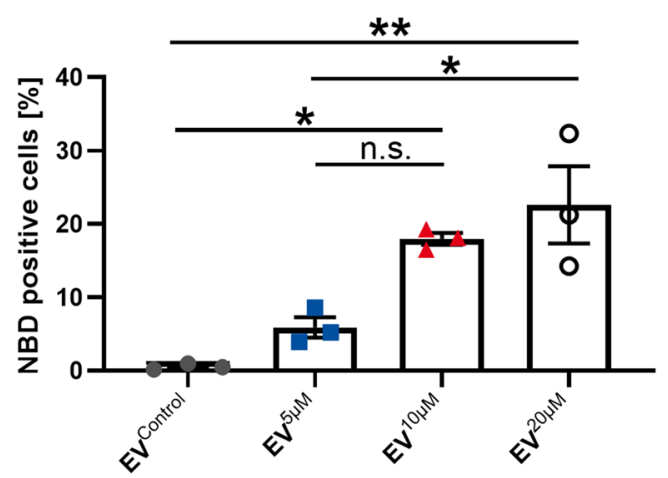


4Fig. 5 A Chemical structure of C16 ceramide and NBD-C12 ceramide. B Fluorescence microscopic imaging of NBD-C12 ceramide (green)-treated HCAECs, after counterstaining with DAPI (blue). C Upper row: Fluorescence microscopic imaging of HCAECs after uptake of $1 \mathrm{EV}$ s from NBD-C12 ceramide-treated HCAECs, after counterstaining with DAPI. Lower row: Imaging of $1 E V$-treated HCAECs, with prior lysis of the EVs in $1 \%$ Triton-X. D+E Absolute and relative quantification of fluorescent $1 E V$-uptake in HCAECs, $n=3$. All data are presented as individual experiments with the mean \pm SEM; $* p<0.05, * * p<0.01$, ANOVA + Bonferoni's multiple comparison test

was mirrored by a significantly elevated enzyme activity of nSMase and protein expression in the cellular lysate, while acidic sphingomyelinase activity remained unchanged (Fig. 2B-D). As the vesicular export of C16 ceramide (d18:1-16:0) was most significantly changed by glucose injury, we also investigated, if the expression of ceramide synthase 5 and 6 (CerS5 + CerS6) would be altered, because these two enzymes govern the de novo synthesis of C16 ceramide. No differences were detected in CerS5 or CerS6 expression after $72 \mathrm{~h}$ of hyperglycemic injury in HCAECs (Figure S3A). This indicates that in our model the sphingomyelinase pathway is activated, whereas expression of the de novo synthesis pathway remains unchanged. The IEVs from HCAECs after hyperglycemic injury were then used to treat native HCAECs, to investigate the effect of the glucose treatment on the ability of the $1 \mathrm{EV}$ s to change the phenotype of a recipient cell. What we found was that the ceramide rich IEVs from hyperglycemic HCAECs significantly reduced viability and increased apoptosis in $1 \mathrm{EV}$-treated HCAECs (Fig. 2E+F). Prior degradation of the EVs with Triton-X completely abrogated the pro-apoptotic effect of the EVs (Figure S4). To exclude that the observed effects of glucose on SMPD3 expression and apoptosis induction in IEV recipient cells were due to unspecific osmotic stress, we repeated the key experiments with a mannitol supplement of $30 \mathrm{mM}$ to have identical osmotic conditions. Mannitol treatment neither reduced HCAEC viability, nor induced apoptosis in EV-recipient cells (Fig. 2G+H). Similarly, the mRNA expression levels of sphingomyelinases or ceramide synthases remained unchanged after osmotic injury with mannitol (Figure S3B). This result indicates, that the observed effect of glucose on SMPD3 activation and apoptosis induction through vesicular ceramide transfer is independent from osmotic injury.

\section{IEVs from hyperglycemic mice induce apoptosis in MCAECs}

To confirm the pro-apoptotic effect of IEVs from hyperglycemic conditions, we performed an ex vivo experiment, for which we induced hyperglycemia in mice through the injection of STZ. From those mice, lEVs were isolated by differential centrifugation and characterized by NTA and immunoblotting, which confirmed a comparable size distribution as for the HCAEC-derived EVs and the presence of Flotillin-1, whereas the nuclear protein Histone 3 was absent in the murine IEVs (Fig. 3A-D). The STZ-treated mice were confirmed to be hyperglycemic, 21 days after the injection (Fig. 3E). Incubation of MCAECs with murine lEVs from hyperglycemic mice led to significantly higher levels of apoptosis than incubation with $\mathrm{lEVs}$ from normoglycemic mice or no lEVs (Fig. 3F). No difference in the levels of IEVs in the blood was detected after 21 days of STZ treatment (Fig. 3G).

\section{Externally applied ceramide is exported in IEVs and induces apoptosis in IEV recipient cells}

To test the hypothesis, that increased ceramide levels in $\mathrm{IEVs}$ induce apoptosis in $\mathrm{IEV}$ - recipient HCAECs, we generated $1 E V s$ with artificially increased ceramide levels. To this end, we used purified C16 ceramide and applied it externally in cell culture at $20 \mu \mathrm{M}$ for $24 \mathrm{~h}$. The cellular uptake and packaging of the $\mathrm{C} 16$ ceramide into lEVs was confirmed by mass spectrometry (Fig. 4A). MTT viability assay and flow-cytometric analysis of the HCAECs themselves showed that exogenous $\mathrm{C} 16$ ceramide is a potent inductor of apoptosis and reduces cell viability in a dosedependent manner (Fig. 4B + C). There was no effect of $\mathrm{C} 16$ treatment on $1 \mathrm{EV}$ release or size distribution, as shown with nanoparticle tracking analysis (Fig. 4D+E). The $1 E V$ s released from cells treated with $\mathrm{C} 16$ ceramide reduced viability and increased apoptosis in the recipient HCAECs, also in a dose-dependent manner, with $10 \mu \mathrm{M}$ C16 leading to $14.6 \%$ apoptosis and $20 \mu \mathrm{M} \mathrm{C} 16$ to $16.2 \%$ apoptosis in the recipient cells (Fig. 4F + G). Furthermore, caspase 3/7 activity was assessed in HCAECs after treatment with C16 ceramide-enriched 1EVs (Figure S5A). Additionally, a proteome profiler array confirmed, the activation of caspase 3 and revealed increased levels of FADD, p27, and phospho-p53 in HCAECs after treatment with $\mathrm{lEV}^{20 \mu \mathrm{MC} 16}$ (Figure S5B).

\section{IEVs are effective transporters of ceramides between HCAECs}

Next, we sought to evaluate if lEVs can effectively transfer ceramides between HCAECs. Therefore, we used a fluorescently labeled ceramide (NBD-C12 ceramide). NBD-C12 ceramide was selected because, owing to the NBD group, it has a similar acyl chain length as native C16 ceramide, which was the most abundant ceramide 
(A)

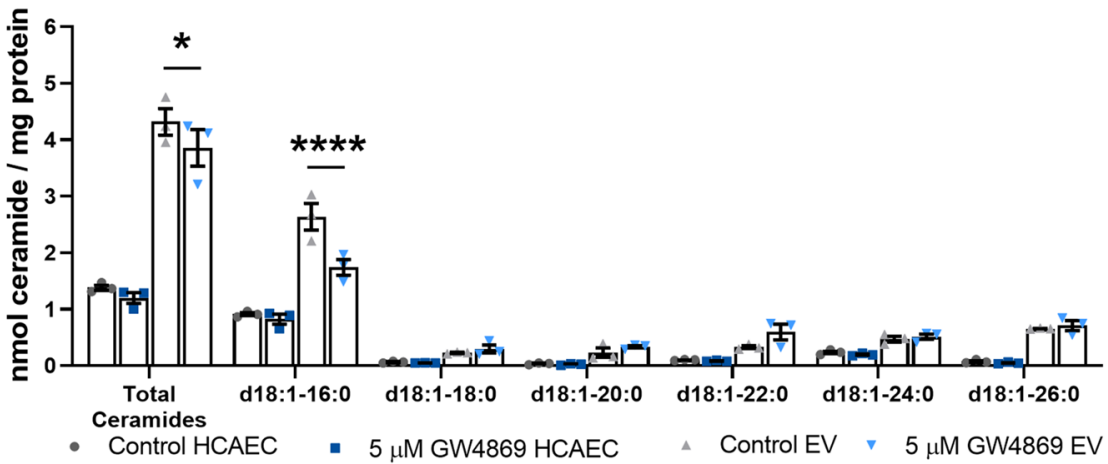

(B)

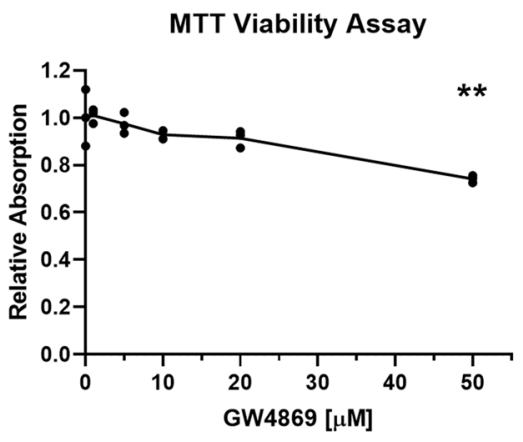

(D)

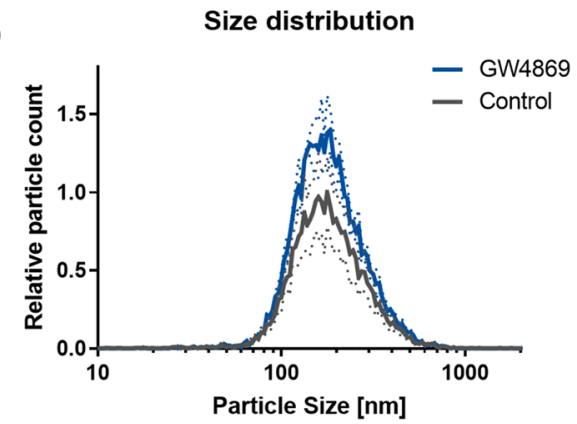

(F) Apoptosis induction after IEV treament

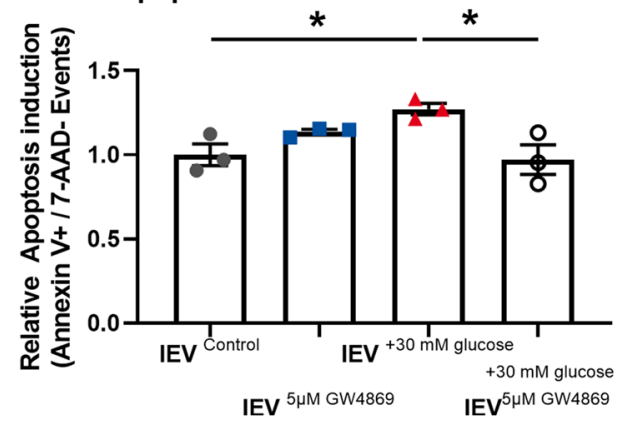

(C) Apoptosis induction after GW4869 treament

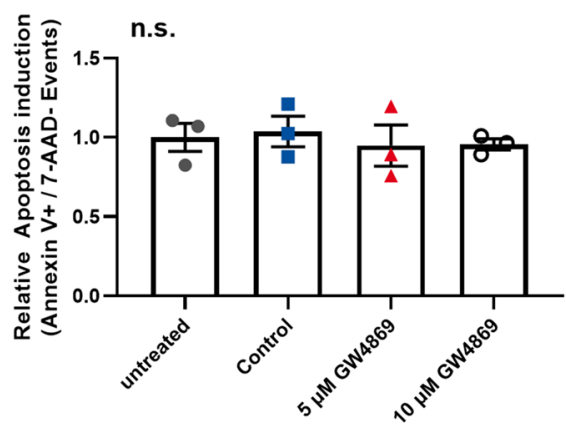

(E)

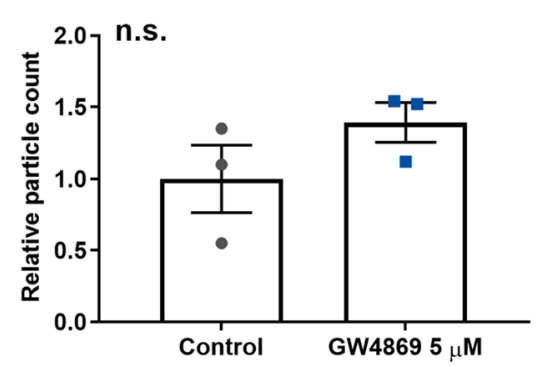

(G)

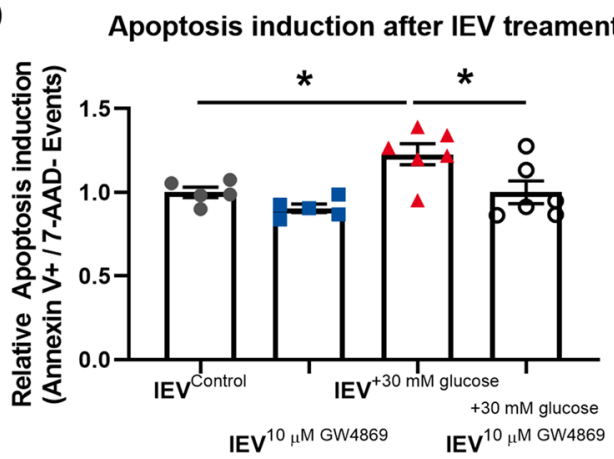

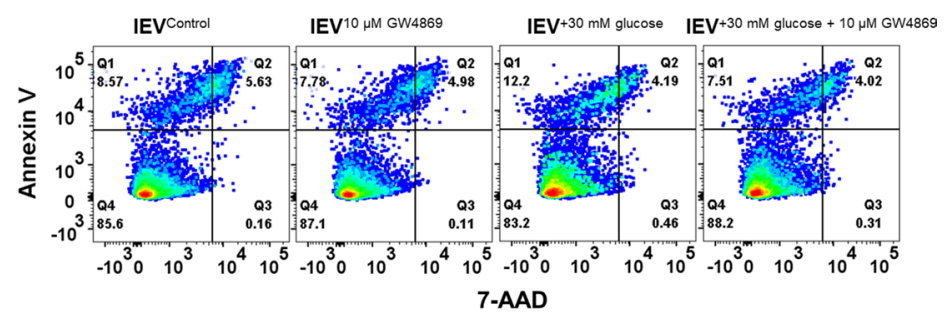


4Fig. 6 A Mass spectrometric analysis of ceramides in HCAECs and IEVs after treatment with $5 \mu \mathrm{M}$ GW4869 for $24 \mathrm{~h}, n=3$. B MTT viability assay in HCAECs after treatment with GW4869 for $24 \mathrm{~h}$, $n=2-3$. C Flow-cytometric analysis of the induction of apoptosis in HCAECs after treatment with GW4869 for $24 \mathrm{~h}, n=3$. D + E Distribution of $1 E V$ sizes and relative $1 \mathrm{EV}$ release after treatment of HCAECs with $5 \mu \mathrm{M}$ GW4869 for $24 \mathrm{~h}$, as analyzed by nanoparticle tracking analysis, $n=3$. $\mathbf{F}+\mathbf{G}$ Flow-cytometric analysis of the induction of apoptosis in HCAECs that were treated with $\mathrm{lEV}$ s from HCAECs after glucose injury and/or $5 \mu \mathrm{M}$ or $10 \mu \mathrm{M}$ GW4869 treatment, (below) representative dot blots, $n=3 / n=5-6$. All data are presented as individual experiments with the mean \pm SEM; n.s. not significant, $* p<0.05, * * p<0.01, * * * * p<0.0001$. An unpaired $t$-test was used for E, ANOVA + Bonferoni's multiple comparison test were used for B, C, F, G, 2-way ANOVA + Bonferoni's multiple comparison test were used for $\mathbf{A}$

in HCAEC-derived lEVs (Fig. 5A). Effective uptake of NBD-C12 ceramide was achieved at doses of $10 \mu \mathrm{M}$ and $20 \mu \mathrm{M}$ after $24 \mathrm{~h}$, as shown by fluorescence microscopy. Notably, NBD-C12 ceramide was shown to have a perinuclear distribution, indicating that intracellular localization was achieved (Fig. 5B). lEVs were isolated from NBD-C12 ceramide-treated HCAECs and incubated with native HCAECs for $12 \mathrm{~h}$. In this case, the transfer of NBDC12 ceramide was observed by fluorescence microscopic imaging. Intracellular NBD-C12 ceramide was detected in the perinuclear region of the recipient cells, similar to the distribution observed after direct exposure of the cells to NBD-C12 ceramide (Fig. 5C). We found the uptake of fluorescent lEVs by recipient cells to be dependent on the dose of NBD-C12 used to treat the lEV-releasing HCAECs, with $5 \mu \mathrm{M}$ NBD-C12 leading to $5.9 \%, 10 \mu \mathrm{M}$ leading to $17.6 \%$, and $20 \mu \mathrm{M}$ leading to $22.6 \%$ NBD-positive cells (Fig. 5D+E). Degradation of the lEVs by $1 \%$ Triton-X completely abolished the transfer of ceramide. Spontaneous formation of NBD-C12 micelles in an aqueous solution, that are co-isolated with lEVs, was excluded using differential centrifugation of NBD-C12 containing PBS with subsequent incubation of the isolated "pseudoEVs" with native HCAECs. No noticeable uptake of NBDC12 "pseudoEVs" was detected (Figure S6). These experiments suggest that ceramides can be transferred between HCAECs through lEVs.

\section{Pharmacological inhibition of nSMase activity reduces vesicular ceramide levels and counteracts the induction of apoptosis in IEV recipient cells}

To confirm that packaging of ceramides into lEVs is SMase dependent, we treated HCAECs with GW4869, a pharmacological inhibitor of nSMase2. Mass spectrometric analysis of GW4869-treated IEVs revealed a significant reduction in the total ceramide levels, which was mainly driven by a reduction in $\mathrm{C} 16$ ceramide, again found to be the most abundant ceramide (Fig. 6A). Cell viability was not significantly reduced at concentrations of GW4869 below $20 \mu \mathrm{M}$ and GW4869 did not cause a direct induction of apoptosis (Fig. $6 \mathrm{~B}+\mathrm{C}$ ). Treatment of cells with $5 \mu \mathrm{M}$ GW4869 showed a tendency towards increased IEV release, but the change was not significant (Fig. 6D+E). The combination of GW4869 with hyperglycemic injury abolished the induction of apoptosis in lEV-recipient cells at concentrations of $5 \mu \mathrm{M}$ GW4869 and $10 \mu \mathrm{M}$ GW4869 (Fig. 6F+G). Additionally, we investigated how glucose injury and GW4869 treatment affects the release of sEVs as well as the ability of sEVs to induce apoptosis in HCAEC-recipient cells. We found that hyperglycemia-induced SMPD3 activation increases SEV release, which could be counteracted by GW4869 treatment (Figure S7A+B). Neither hyperglycemia nor GW4869 treatment significantly changed apoptosis induction in endothelial sEV-recipient cells (Figure S7D).

\section{SiRNA-mediated downregulation of SMPD3 counteracts apoptosis induction after hyperglycemic injury}

Additionally, we sought to determine whether the vesicular transfer of ceramides was dependent on nSMase 2 activity in the EV-releasing HCAECs. Therefore, we performed siRNA-mediated downregulation of SMPD3. The transfection efficiency of the siRNAs was confirmed via qPCR under normal and hyperglycemic conditions (Fig. 7A+B). The viability of the HCAECs was shown to be unchanged after transfection of the siRNAs (Fig. 7C). Downregulation of SMPD3 led to a significant increase in $\mathrm{IEV}$ release (Fig. 7D+E). Similar to the pharmacological inhibition by GW4869, siRNA-mediated downregulation of SMPD3 in combination with hyperglycemic injury abolished the induction of apoptosis in HCAECs that take up lEVs (Fig. 7E+F).

\section{Discussion}

In the present manuscript, we show for the first time that hyperglycemic injury enhances ceramide transfer in lEVs between endothelial cells. Increased packaging of ceramide in the lEVs induces apoptosis in endothelial lEV-recipient cells. Hyperglycemia activates nSMase2, which controls the pro-apoptotic function of IEVs after hyperglycemic injury.

The involvement of nSMase 2 in the release of small EVs was discovered more than a decade ago [55, 56]. Consequently, the nSMase inhibitor GW4869 has been used in various studies as an inhibitor of the release of small EVs $[57,58]$ and has even found its way into the 2018 MISEV guidelines as an "exosome inhibitor" [54]. The effect of nSMase2 inhibition on IEV release, however, remained controversial. In an early study, inhibitors of nSMase 2 did 

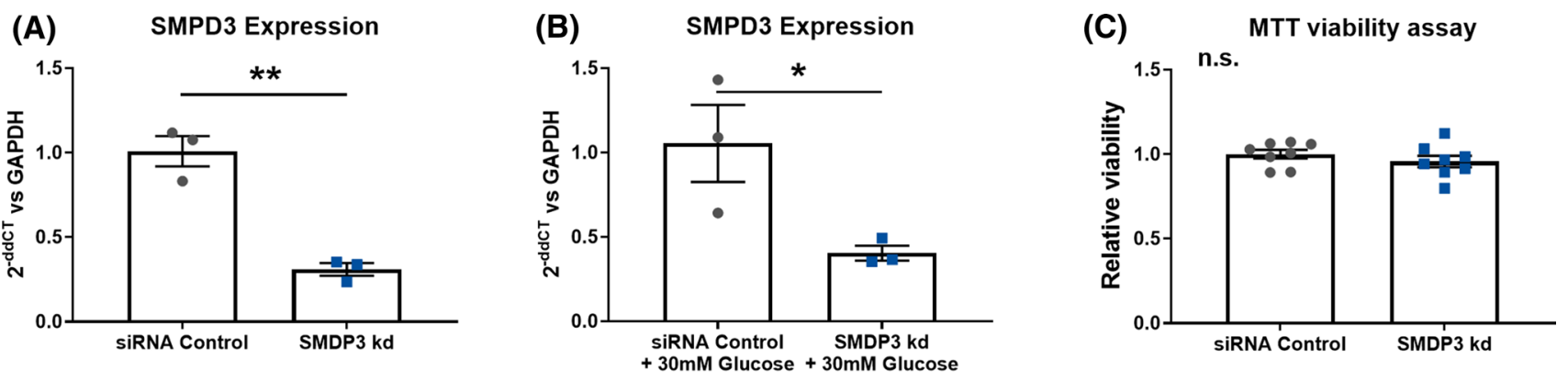

(D)

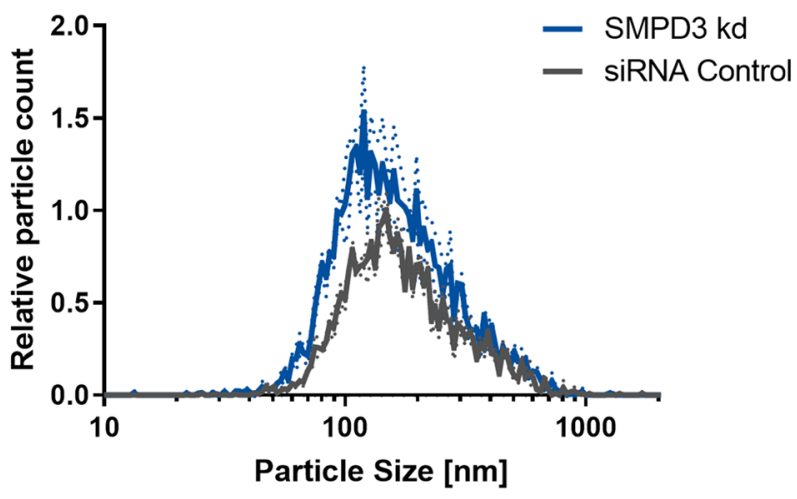

(F) Apoptosis induction after IEV treatment
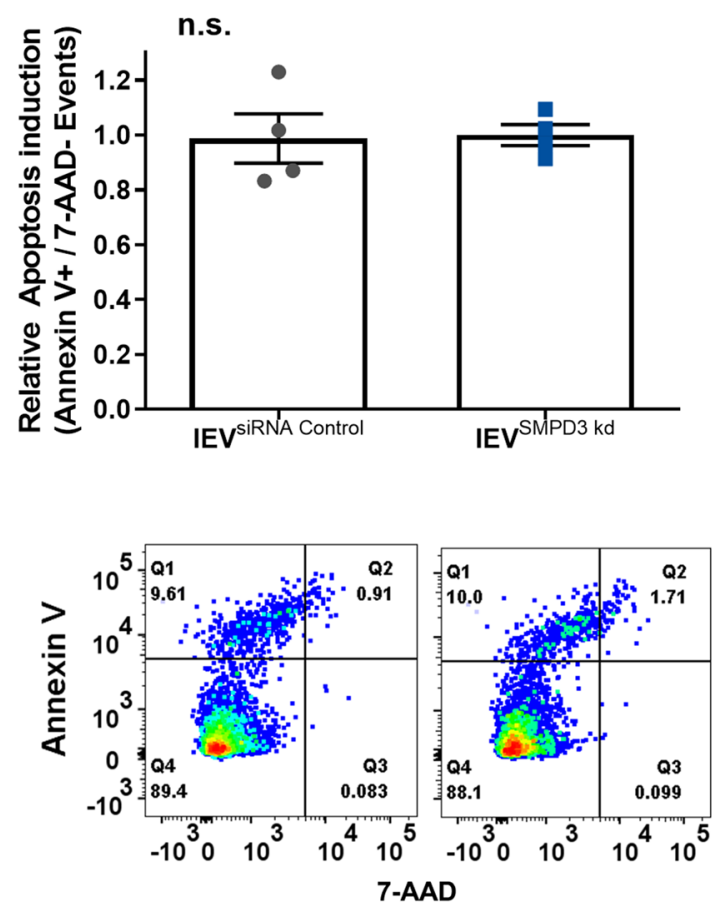

(E)

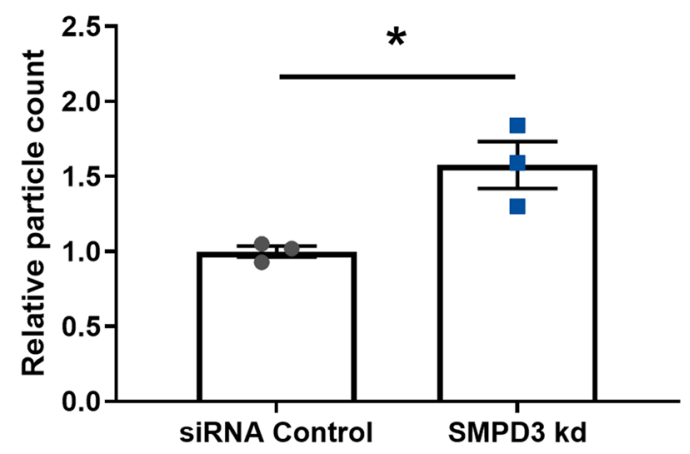

(G)

Apoptosis induction after IEV treatment
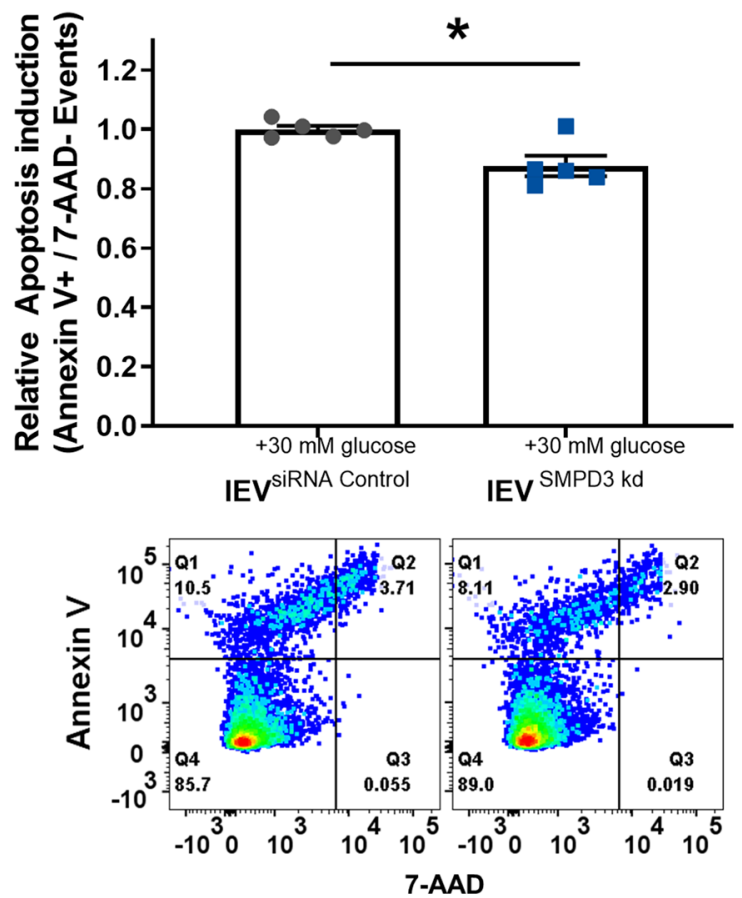
4Fig. 7 A + B Confirmation of the effective downregulation of SMPD3 with siRNA in HCAECs under normal and hyperglycemic conditions, $n=3$. C MTT viability assay in HCAECs after downregulation of $S M P D 3, n=6$. D $+\mathbf{E}$ Distribution of $1 E V$ sizes and relative IEV release after downregulation of SMPD3, as analyzed by nanoparticle tracking analysis, $n=3$. F + G Flow-cytometric analysis of the induction of apoptosis in HCAECs that were treated with IEVs from HCAECs after downregulation of SMPD3 and glucose injury, (below) representative dot blots, $n=4 / n=6$. All data are presented as individual experiments with the mean $\pm \mathrm{SEM}$; n.s. not significant, ${ }^{*} p<0.05, * * p<0.01$. An unpaired t-test was used for $\mathbf{A}-\mathbf{G}$

not significantly affect the release of $1 \mathrm{EV}$ s from glial cells [59], whereas Menck et al. could show that inhibition of SMPD2 and SMPD3 gene expression as well as inhibition of nSMase activity through GW4869 enhanced lEV release from a breast cancer cell line [30]. In our study, we saw that siRNA-mediated downregulation of SMPD3 increases IEV release from endothelial cells, which also seemed to be the case after GW4869 treatment (although borderline not significant). In contrast, enhanced nSMase 2 activity after glucose injury only caused a non-significant trend towards reduced release of $1 \mathrm{EV}$ s. nSMase 2 therefore mainly controls $1 \mathrm{EV}$ ceramide export in endothelial cells and only has a minor inhibitory effect on $1 E V$ release.

As with all lipids, the route of delivery plays an important role in the biological activity of ceramides. Ceramides that are delivered in liposomes or other complexes are several times more potent than uncomplexed, externally applied ceramides [60-62]. In our experiments, we showed that the ceramide transfer via vesicles is more effective than external application in DMSO. For the experiment in Fig. 4C, with $10 \mu \mathrm{M}$ ceramide in 2-ml culture medium, a total of $20 \mathrm{nmol}$ C16 ceramide were used. For the experiment in Fig. 4G, we can extrapolate from the mass spectrometric analysis in Fig. 4A that the EVs from $2.0 \times 10^{6} \mathrm{HCAECs}$ after stimulation with $20 \mu \mathrm{M}$ ceramide as used for stimulation experiments carry 28.4 pmol $\mathrm{C} 16$ and 88.5 pmol total ceramide. Therefore, lEV delivery of ceramides seems more effective than applying it extracellularly. For small EVs, intercellular ceramide transfer has been assessed in the context of inflammatory liver disease and neurodegenerative disease [63-65]: For inflammatory liver disease, the authors found that hepatocytes release ceramide-rich small EVs after injury with palmitate, which triggered macrophage recruitment through chemotaxis [64]. Furthermore, ceramide-rich exosomes were shown to contribute to amyloid-induced apoptosis and to be involved in the induction of apoptosis in oligodendrocytes $[63,65]$. Our work expands these findings to $1 \mathrm{EVs}$ and to the spectrum of diabetes-induced cardiovascular disease. Of note, the induction of apoptosis after C16 ceramide treatment was more pronounced than after hyperglycemic injury, which might additionally increase the packaging of other pro-apoptotic molecules. This was not assessed in the current study. Additionally, we cannot rule out that activation of nSMase 2 affects the composition of $1 \mathrm{EVs}$ beyond the ceramide content of the $\mathrm{IEVs}$, which could also explain the alteration in the induction of apoptosis in $\mathrm{lEV}$-recipient cells. Considering the complex composition of $1 E V s$, including various kinds of nucleic acids, proteins, peptides, and lipids other than sphingolipids, this question will require futher studies to be answered definitively.

In our study, the ceramide/protein ratio is higher in lEVs than in the respective EV-releasing cells (Fig. 1H). This observation confirms the results of a study from Haraszti et al., who showed that microvesicles (pelleted at $10,000 \times g$ ) from various cell types have higher ceramide/protein ratios than the respective mother cells, but also higher than exosomes, which had an even lower ceramide/protein ratio than their mother cells [27]. Thus, $1 E V$ s are potentially more effective intercellular transporters of ceramides than small EVs. Although the size distribution of our EVs does not suggest that there are large numbers of apoptotic bodies present in the samples, their presence cannot be excluded entirely, due to a lack of specific markers for apoptotic bodies.

Severe hyperglycemia ( $>33 \mathrm{mmol} / \mathrm{L}$ glucose), which causes a hyperglycemic crisis, is a severe, but not rare, complication of diabetes mellitus [40]. Consequently, the addition of $30 \mathrm{mmol} / \mathrm{L}$ glucose to the culture medium is a realistic and pathophysiologically relevant model. A reduction in endothelial cell viability of $15-20 \%$ can therefore be considered a severe effect, which may contribute to the high mortality of patients with a hyperglycemic crisis $[66,67]$. In accordance with our study, hyperglycemia has previously been reported to induce endothelial-cell apoptosis [19, 36, 68-70]. The implication of nSMase2 and ceramides in this process remains controversial. In contrast to our study, Ido et al. reported to have found no evidence for activation of ceramide producing enzymes in hyperglycemic endothelial cells [36]. This is, in our opinion, due to two key differences in the study design: First, the glucose treatment in the study by Ido and co-workers only lasted $24 \mathrm{~h}$, in contrast to $72 \mathrm{~h}$ in our study, and second, no in-depth analysis of cellular enzyme activities was performed. A more recent study found increased aSMase activity after only $24 \mathrm{~h}$ of hyperglycemic injury, while again the nSMase activity was not assessed [69]. Another study reported that a reduction of the cellular ceramide levels by overexpression of acid ceramidase alleviates glucose-induced apoptosis in endothelial cells [70]. The involvement of nSMase 2 in this process has not yet been reported, although ceramide accumulation is generally a well-known inductor of endothelial cell apoptosis [1, 71].

Independent of hyperglycemic injury, cellular ceramide accumulation is a strong pro-apoptotic and proinflammatory signal that involves the activation of p53 and the NRLP3 inflammasome [72, 73]. Furthermore, high 
levels of nSMase 2 activity mediate TNF $\alpha$-induced inflammatory processes [74]. These pathways are known to be master regulators of atherosclerotic plaque development, in which ceramides therefore contribute to the pro-inflammatory milieu [6]. Consequently, inhibition of nSMase2 is a potential target to counteract pro-inflammatory signaling in these cells [11]. In the context of cardiovascular disease, the plasma levels of long-chain ceramides have been linked to adverse events after coronary angiography [1] and cardiovascular death from coronary artery disease [2]. Vesicular ceramide levels have, however, been underappreciated in recent research. Therefore, the potential for vesicular ceramide levels to serve as biomarkers of acute myocardial infarction has only been very recently discovered [35]. Our work may contribute to this direction and highlights the importance of vesicular ceramide transfer as a mediator of diabetes-associated cardiovascular disease development.

In summary, we found evidence of a new cellular mechanism for the induction of endothelial-cell apoptosis under hyperglycemic conditions. We identified the inhibition of nSMase 2 activity as a promising approach to therapeutically influence this mechanism. Additionally, we established a role for $1 \mathrm{EV}$ s as efficient intercellular transport vehicles for ceramides.

Supplementary Information The online version contains supplementary material available at https://doi.org/10.1007/s00018-021-04049-5.

Acknowledgements The authors thank Paula Levermann, Inga Titova, Anna Flender, and Sarah Arahouan for excellent technical support. We thank Meghan Lucas for her critical reading and valuable suggestions to improve the manuscript. We thank Felix Babatz and Astrid Schauss from the Imaging Facility-CECAD Cologne for help with the electron microscopic imaging. We would like to thank Katrin S. Reiners from the Institute of Clinical Chemistry and Clinical Pharmacology, University of Bonn for providing the Zetaview NTA. We thank Benjamin Krämer from the Medical Clinic I, Internal Medicine, University Hospital Bonn for guidance and access to the flow cytometer. We thank Helga Peisker from IMBIO, University of Bonn for help with the operation of the Q-Trap mass spectrometer.

Author contributions Conceptualization: AZ, FJ, NW, GN; investigation: AZ, ALJ, KG, MB; methodology: AZ, ALJ, MB, PDue, MRH, KG; data curation: $A Z, A L J, M B, K G$, PDue, MRH; supervision: PDoe, FJ, NW, GN; funding: AZ, ALJ, NW, FJ; manuscript writing: AZ; manuscript review and editing: ALJ, KG, PDoe, MB, PDue, MRH, NW, GN, FJ.

Funding Open Access funding enabled and organized by Projekt DEAL. This work was supported by the medical faculty of the University of Bonn (BONFOR Grant No. 2018-1A-07 and 2020-2A-05 to A.Z.), the German Heart Foundation (to A.L.J.), the German Cardiac Society (DGK 16/2018 to A.Z.), the Ernst \& Berta Grimmke Foundation (13/19 to A.Z.), the Deutsche Forschungsgemeinschaft (WE 4139/8-1 to N.W., JA 2351/2-1 to F.J., and Project-ID 397484323 TRR 259 to F.J.), and the Corona-Foundation (to F.J.).
Availability of data and materials All data are available from the corresponding author upon reasonable request.

\section{Declarations}

Conflict of interest The authors declare that no competing interests exist.

Open Access This article is licensed under a Creative Commons Attribution 4.0 International License, which permits use, sharing, adaptation, distribution and reproduction in any medium or format, as long as you give appropriate credit to the original author(s) and the source, provide a link to the Creative Commons licence, and indicate if changes were made. The images or other third party material in this article are included in the article's Creative Commons licence, unless indicated otherwise in a credit line to the material. If material is not included in the article's Creative Commons licence and your intended use is not permitted by statutory regulation or exceeds the permitted use, you will need to obtain permission directly from the copyright holder. To view a copy of this licence, visit http://creativecommons.org/licenses/by/4.0/.

\section{References}

1. Meeusen JW et al (2018) Plasma ceramides: a novel predictor of major adverse cardiovascular events after coronary angiography. Arterioscler Thromb Vasc Biol 38:1933-1939

2. Laaksonen $\mathrm{R}$ et al (2016) Plasma ceramides predict cardiovascular death in patients with stable coronary artery disease and acute coronary syndromes beyond LDL-cholesterol. Eur Heart J 37(25):1967-1976

3. Hilvo M et al (2019) Development and validation of a ceramideand phospholipid-based cardiovascular risk estimation score for coronary artery disease patients. Eur Heart J. https://doi.org/10. 1093/eurheartj/ehz387

4. Mullen TD, Obeid LM (2012) Ceramide and apoptosis: exploring the enigmatic connections between sphingolipid metabolism and programmed cell death. Anticancer Agents Med Chem 12(4):340-363

5. Aflaki E et al (2012) C16 ceramide is crucial for triacylglycerolinduced apoptosis in macrophages. Cell Death Dis 3:e280

6. Edsfeldt A et al (2016) Sphingolipids contribute to human atherosclerotic plaque inflammation. Arterioscler Thromb Vasc Biol 36(6): 1132-1140

7. Shamseddine AA, Airola MV, Hannun YA (2015) Roles and regulation of neutral sphingomyelinase-2 in cellular and pathological processes. Adv Biol Regul 57:24-41

8. Wegner MS et al (2016) The enigma of ceramide synthase regulation in mammalian cells. Prog Lipid Res 63:93-119

9. Airola MV et al (2017) Structure of human nSMase2 reveals an interdomain allosteric activation mechanism for ceramide generation. Proc Natl Acad Sci USA 114(28):E5549-E5558

10. Kim MY et al (1991) Identification of sphingomyelin turnover as an effector mechanism for the action of tumor necrosis factor alpha and gamma-interferon. Specific role in cell differentiation. J Biol Chem 266(1):484-489

11. Lallemand $\mathrm{T}$ et al (2018) nSMase2 (Type 2-neutral sphingomyelinase) deficiency or inhibition by GW4869 reduces inflammation and atherosclerosis in Apoe. Arterioscler Thromb Vasc Biol 38(7):1479-1492

12. Chaurasia B et al (2019) Targeting a ceramide double bond improves insulin resistance and hepatic steatosis. Science 365(6451):386-392 
13. Schramm TK et al (2008) Diabetes patients requiring glucoselowering therapy and nondiabetics with a prior myocardial infarction carry the same cardiovascular risk: a population study of 3.3 million people. Circulation 117(15):1945-1954

14. Gregg EW, Williams DE, Geiss L (2014) Changes in diabetes-related complications in the United States. N Engl J Med 371(3):286-287

15. Jansen $\mathrm{F}$ et al (2017) Endothelial- and immune cell-derived extracellular vesicles in the regulation of cardiovascular health and disease. JACC Basic Transl Sci 2(6):790-807

16. Davignon J, Ganz P (2004) Role of endothelial dysfunction in atherosclerosis. Circulation 109(23 Suppl 1):III27-III32

17. Zhang QJ et al (2012) Ceramide mediates vascular dysfunction in diet-induced obesity by PP2A-mediated dephosphorylation of the eNOS-Akt complex. Diabetes 61(7):1848-1859

18. Ceriello A et al (1996) High glucose induces antioxidant enzymes in human endothelial cells in culture. Evidence linking hyperglycemia and oxidative stress. Diabetes 45(4):471-477

19. Guan $\mathrm{G}$ et al (2014) Neferine prevented hyperglycemia-induced endothelial cell apoptosis through suppressing ROS/Akt/NF- $\kappa \mathrm{B}$ signal. Endocrine 47(3):764-771

20. Li H et al (2002) Dual effect of ceramide on human endothelial cells: induction of oxidative stress and transcriptional upregulation of endothelial nitric oxide synthase. Circulation 106(17):2250-2256

21. Hergenreider E et al (2012) Atheroprotective communication between endothelial cells and smooth muscle cells through miRNAs. Nat Cell Biol 14(3):249-256

22. Nozaki T et al (2009) Significance of a multiple biomarkers strategy including endothelial dysfunction to improve risk stratification for cardiovascular events in patients at high risk for coronary heart disease. J Am Coll Cardiol 54(7):601-608

23. Sinning JM et al (2011) Circulating CD31+/Annexin V+ microparticles correlate with cardiovascular outcomes. Eur Heart J 32(16):2034-2041

24. Hutcheson JD et al (2016) Genesis and growth of extracellularvesicle-derived microcalcification in atherosclerotic plaques. Nat Mater 15(3):335-343

25. Deatherage BL, Cookson BT (2012) Membrane vesicle release in bacteria, eukaryotes, and archaea: a conserved yet underappreciated aspect of microbial life. Infect Immun 80(6):1948-1957

26. Yáñez-Mó M et al (2015) Biological properties of extracellular vesicles and their physiological functions. J Extracell Vesicles 4:27066

27. Haraszti RA et al (2016) High-resolution proteomic and lipidomic analysis of exosomes and microvesicles from different cell sources. J Extracell Vesicles 5:32570

28. Trajkovic $\mathrm{K}$ et al (2008) Ceramide triggers budding of exosome vesicles into multivesicular endosomes. Science 319(5867):1244-1247

29. Verderio C, Gabrielli M, Giussani P (2018) Role of sphingolipids in the biogenesis and biological activity of extracellular vesicles. J Lipid Res 59(8):1325-1340

30. Menck K et al (2017) Neutral sphingomyelinases control extracellular vesicles budding from the plasma membrane. J Extracell Vesicles 6(1):1378056

31. Serban KA et al (2016) Structural and functional characterization of endothelial microparticles released by cigarette smoke. Sci Rep 6:31596

32. Zietzer A et al (2020) MicroRNA-mediated vascular intercellular communication is altered in chronic kidney disease. Cardiovasc Res. https://doi.org/10.1093/cvr/cvaa322

33. Loyer X et al (2014) Microvesicles as cell-cell messengers in cardiovascular diseases. Circ Res 114(2):345-353
34. New SE et al (2013) Macrophage-derived matrix vesicles: an alternative novel mechanism for microcalcification in atherosclerotic plaques. Circ Res 113(1):72-77

35. Burrello J et al (2020) Sphingolipid composition of circulating extracellular vesicles after myocardial ischemia. Sci Rep 10(1): 16182

36. Ido Y, Carling D, Ruderman N (2002) Hyperglycemia-induced apoptosis in human umbilical vein endothelial cells: inhibition by the AMP-activated protein kinase activation. Diabetes 51(1):159-167

37. Jansen F et al (2017) Endothelial microparticle-promoted inhibition of vascular remodeling is abrogated under hyperglycaemic conditions. J Mol Cell Cardiol 112:91-94

38. Jansen F et al (2013) Endothelial microparticle-mediated transfer of MicroRNA-126 promotes vascular endothelial cell repair via SPRED1 and is abrogated in glucose-damaged endothelial microparticles. Circulation 128(18):2026-2038

39. Pieper GM, Dondlinger L (1997) Glucose elevations alter bradykinin-stimulated intracellular calcium accumulation in cultured endothelial cells. Cardiovasc Res 34(1):169-178

40. Benoit SR et al (2020) Trends in emergency department visits and inpatient admissions for hyperglycemic crises in adults with diabetes in the U.S., 2006-2015. Diabetes Care 43(5):1057-1064

41. Crewe $\mathrm{C}$ et al (2018) An endothelial-to-adipocyte extracellular vesicle axis governed by metabolic state. Cell 175(3):695-708.e13

42. Zietzer A et al (2020) The RNA-binding protein hnRNPU regulates the sorting of microRNA-30c-5p into large extracellular vesicles. J Extracell Vesicles 9(1):1786967

43. Hromada C et al (2017) Endothelial extracellular vesicles-promises and challenges. Front Physiol 8:275

44. Chandler WL (2016) Measurement of microvesicle levels in human blood using flow cytometry. Cytometry B Clin Cytom 90(4):326-336

45. Menck K et al (2017) Isolation and characterization of microvesicles from peripheral blood. J Vis Exp. https://doi.org/10.3791/ 55057

46. Jamaly $\mathrm{S}$ et al (2018) Impact of preanalytical conditions on plasma concentration and size distribution of extracellular vesicles using Nanoparticle Tracking Analysis. Sci Rep 8(1):17216

47. Liu $\mathrm{Y}$ et al (2018) Atherosclerotic conditions promote the packaging of functional MicroRNA-92a-3p into endothelial microvesicles. Circ Res 124:575-587

48. Hosen MR et al (2021) CAD increases the long noncoding RNA PUNISHER in small extracellular vesicles and regulates endothelial cell function via vesicular shuttling. Mol Ther Nucleic Acids 25:388-405

49. Ginkel C et al (2012) Ablation of neuronal ceramide synthase 1 in mice decreases ganglioside levels and expression of myelin-associated glycoprotein in oligodendrocytes. J Biol Chem 287(50):41888-41902

50. Wewer V et al (2011) Quantification of sterol lipids in plants by quadrupole time-of-flight mass spectrometry. J Lipid Res 52(5):1039-1054

51. Woeste MA et al (2019) Species-specific differences in nonlysosomal glucosylceramidase GBA2 function underlie locomotor dysfunction arising from loss-of-function mutations. J Biol Chem 294(11):3853-3871

52. Brosius F (2015) Diabetic Complications Consortium: HighDose Streptozotocin Induction Protocol (Mouse). https://www. diacomp.org/shared/document.aspx?id=74\&docType=Protocol.

53. Berglund ED et al (2008) Glucose metabolism in vivo in four commonly used inbred mouse strains. Diabetes 57(7):1790-1799

54. Théry C and Witwer KW (2018) Minimal Information for Studies of Extracellular Vesicles 2018 (MISEV 2018): a position statement of the International Society for Extracellular Vesicles and 
update of the MISEV 2014 guidelines. International Society of Extracellular Vesicles

55. Kosaka N et al (2010) Secretory mechanisms and intercellular transfer of microRNAs in living cells. J Biol Chem 285(23): 17442-17452

56. Kosaka $\mathrm{N}$ et al (2013) Neutral sphingomyelinase 2 (nSMase2)dependent exosomal transfer of angiogenic microRNAs regulate cancer cell metastasis. J Biol Chem 288(15):10849-10859

57. Essandoh $\mathrm{K}$ et al (2015) Blockade of exosome generation with GW4869 dampens the sepsis-induced inflammation and cardiac dysfunction. Biochim Biophys Acta 1852(11):2362-2371

58. Catalano M, O'Driscoll L (2020) Inhibiting extracellular vesicles formation and release: a review of EV inhibitors. J Extracell Vesicles 9(1): 1703244

59. Bianco $\mathrm{F}$ et al (2009) Acid sphingomyelinase activity triggers microparticle release from glial cells. EMBO J 28(8):1043-1054

60. Shabbits JA, Mayer LD (2003) Intracellular delivery of ceramide lipids via liposomes enhances apoptosis in vitro. Biochim Biophys Acta 1612(1):98-106

61. Li G et al (2018) Nanoliposome C6-ceramide increases the antitumor immune response and slows growth of liver tumors in mice. Gastroenterology 154(4):1024-1036.e9

62. Kjellberg MA et al (2015) metabolic conversion of ceramides in HeLa cells - a cholesteryl phosphocholine delivery approach. PLoS ONE 10(11):e0143385

63. Podbielska $\mathrm{M}$ et al (2016) Cytokine-induced release of ceramideenriched exosomes as a mediator of cell death signaling in an oligodendroglioma cell line. J Lipid Res 57(11):2028-2039

64. Kakazu E et al (2016) Hepatocytes release ceramide-enriched pro-inflammatory extracellular vesicles in an IRE1 $\alpha$-dependent manner. J Lipid Res 57(2):233-245

65. Wang $\mathrm{G}$ et al (2012) Astrocytes secrete exosomes enriched with proapoptotic ceramide and prostate apoptosis response 4 (PAR-4): potential mechanism of apoptosis induction in Alzheimer disease (AD). J Biol Chem 287(25):21384-21395
66. Pasquel FJ, Umpierrez GE (2014) Hyperosmolar hyperglycemic state: a historic review of the clinical presentation, diagnosis, and treatment. Diabetes Care 37(11):3124-3131

67. Kao Y et al (2016) Subsequent mortality after hyperglycemic crisis episode in the non-elderly: a national population-based cohort study. Endocrine 51(1):72-82

68. Sheu ML et al (2005) High glucose induces human endothelial cell apoptosis through a phosphoinositide 3-kinase-regulated cyclooxygenase- 2 pathway. Arterioscler Thromb Vasc Biol 25(3):539-545

69. Luo Y, Lei M (2017) $\alpha$-Mangostin protects against high-glucose induced apoptosis of human umbilical vein endothelial cells. Biosci Rep. https://doi.org/10.1042/BSR20170779

70. Weikel KA et al (2015) Glucose and palmitate uncouple AMPK from autophagy in human aortic endothelial cells. Am J Physiol Cell Physiol 308(3):C249-C263

71. Bonnaud S et al (2007) Sphingosine-1-phosphate protects proliferating endothelial cells from ceramide-induced apoptosis but not from DNA damage-induced mitotic death. Cancer Res 67(4):1803-1811

72. Fekry B et al (2018) C 16 -ceramide is a natural regulatory ligand of p53 in cellular stress response. Nat Commun 9(1):4149

73. Scheiblich $\mathrm{H}$ et al (2017) Activation of the NLRP3 inflammasome in microglia: the role of ceramide. J Neurochem 143(5):534-550

74. Al-Rashed $\mathrm{F}$ et al (2020) Neutral sphingomyelinase 2 regulates inflammatory responses in monocytes/macrophages induced by TNF- $\alpha$. Sci Rep 10(1):16802

Publisher's Note Springer Nature remains neutral with regard to jurisdictional claims in published maps and institutional affiliations. 